\title{
Heat kernel coefficients on the sphere in any dimension
}

\author{
Yannick Kluth $^{\mathrm{a}}{ }^{\mathbb{D}}$, Daniel F. Litim \\ Department of Physics and Astronomy, University of Sussex, Brighton BN1 9QH, UK
}

Received: 22 November 2019 / Accepted: 25 February 2020 / Published online: 26 March 2020

(C) The Author(s) 2020

\begin{abstract}
We derive all heat kernel coefficients for Laplacians acting on scalars, vectors, and tensors on fully symmetric spaces, in any dimension. Final expressions are easy to evaluate and implement, and confirmed independently using spectral sums and the Euler-Maclaurin formula. We also obtain the Green's function for Laplacians acting on transverse traceless tensors in any dimension, and new integral representations for heat kernels using known eigenvalue spectra of Laplacians. Applications to quantum gravity and the functional renormalisation group, and other, are indicated.
\end{abstract}

\section{Contents}

1 Introduction . . . . . . . . . . . . 1

2 Preliminaries ................. 2

2.1 Heat kernel coefficients . . . . . . . . . . . 2

2.2 Renormalisation group . . . . . . . . . . . 3

3 Heat kernels from Green's functions . . . . . . . . . 4

3.1 Green's function technique . . . . . . . . . . 4

3.2 Scalars . . . . . . . . . . . . . . . 4

3.3 Transverse vectors . . . . . . . . . . . . . 5

3.4 Transverse traceless tensors . . . . . . . . . . . 8

3.5 Unconstrained fields . . . . . . . . . . . . 10

4 Heat kernels from spectral sums . . . . . . . . . . 12

4.1 Spectral sum technique . . . . . . . . . . 12

4.2 Results from Euler-Maclaurin . . . . . . . 13

4.3 Heat kernels from spectral integrals . . . . . . 15

4.4 Analytic continuation . . . . . . . . . . 15

5 Discussion . . . . . . . . . . . 16

Appendix A: Expansion coefficients . . . . . . . . 17

Appendix B: Heat kernels in even dimensions . . . . 18

Appendix C: Spectral integrals . . . . . . . . . . . . . 19

References . . . . . . . . . . . . . . . . 20

a e-mail: y.kluth@sussex.ac.uk (corresponding author)

\section{Introduction}

Heat kernel techniques are well-established tools in both theoretical physics and mathematics [1-5]. They aim at the computation of traces of differential operators, and have a wide range of applications covering fluctuations of quantum fields on curved space-times, ultraviolet divergences and effective actions, spectral functions, quantum anomalies, the Casimir effect, quantum gravity, and more [6-12]. The central idea is to express a certain Green's function as an integral over the so called proper time parameter which satisfies the heat equation. The integrand of this method, the heat kernel, is then a functional of the background metric. While for some special backgrounds it can be calculated exactly, it is not possible to solve it for general manifolds. Approximation schemes have been introduced including the seminal Schwinger-DeWitt expansion giving rise to the heat kernel coefficients. This asymptotic expansion at early proper time works well for small space-time separations, which makes it a convenient tool to study short distance divergences in quantum field theory. On general manifolds, the first six heat kernel coefficients are known [13-21]. Calculations of heat kernel coefficients are greatly simplified on specific manifolds such as maximally symmetric backgrounds where they can be obtained from Green's function [9].

Recent applications of heat kernels and spectral sums on $\mathrm{dS}$ or AdS spaces cover a wide range of activities such as tests of the AdS/CFT correspondence for conformal higher spin models [22,23], derivations of effective actions in supergravity on curved backgrounds [24], and studies of trace anomalies from quantum mechanical path integrals [25], and more. In the context of quantum gravity, heat kernels appear prominently in renormalisation group studies of Hořava gravity [26], and in tests of the asymptotic safety conjecture [27,28], In the latter, specifically, heat kernels on maximally symmetric or Einstein spaces are used to find beta functions for gravitational couplings [28-43]. Intriguingly, results for fixed points and scaling exponents show that the canonical 
mass dimension of couplings remains a good ordering principle, with asymptotically safe quantum gravity becoming "as Gaussian as it gets" [40-43]. Further technical choices $[32,44]$ are commonly adopted to reduce the required heat kernel coefficients on spheres to a finite set [35]. In general, however, the flow of couplings with increasing canonical mass dimension necessitates the knowledge of an increasing number of heat kernel coefficients, many more than presently available.

In this paper, we fill this gap in the literature and compute all heat kernel coefficients for scalars, transverse vectors, and transverse traceless symmetric tensors on fully symmetric backgrounds with Euclidean signature and positive curvature. Our primary input are the known Green's functions for the Laplacian acting on scalars [45] and transverse vectors $[46,47]$. In addition, we derive the Green's function of the Laplacian acting on transverse traceless tensors to find closed expressions for all heat kernel coefficients on spheres, in any dimension. Final expressions are easy to evaluate and implement, and confirmed using spectral sums. We also find new integral representations for heat kernels using known eigenvalue spectra of Laplacians and the Euler-Maclaurin formula. Besides their general interest, our findings enable new tests of the asymptotic safety conjecture without resorting to flat backgrounds or spectral sums and approximations thereof.

The remainder of this paper is organised as follows. In Sect. 2 we recall the definition of heat kernel coefficients from asymptotic expansions (Sect. 2.1) and their usage to calculate functional traces such as in Wilson's renormalisation group (Sect. 2.2). Section 3 contains the main derivation of heat kernel coefficients. After an outline of the method (Sect. 3.1) we compute the heat kernels for scalars (Sect. 3.2), transverse vectors (Sect. 3.3), transverse traceless tensors (Sect. 3.4), and the first coefficients of the asymptotic heat kernel expansion for unconstrained fields (Sect. 3.5). In Sect. 4, we derive heat kernels using spectral sums and the Euler-Maclaurin formula. After an outline of our methodology (Sect. 4.1), we compute heat kernels for selected integer dimensions and confirm our previous findings (Sect. 4.2). We also find new spectral integral representations for heat kernel coefficients (Sect. 4.3) including in general dimensions (Sect. 4.4). Three appendices additionally provide expansion coefficients (Appendix A), heat kernel coefficients in even dimensions (Appendix B), and a derivation of heat kernels from spectral integrals (Appendix C). In Sect. 5 we close with a discussion of results and possible future applications.

\section{Preliminaries}

In this section, we recall basic definitions for heat kernel coefficients and their usage in the context of Wilson's renormal- isation group. Ultimately, we aim at finding the heat kernel expansion for different fields on the sphere using their corresponding Green's functions. Thus, from now on we focus on the specific case of a fully symmetric background manifold, even though some of the considerations are more general.

\subsection{Heat kernel coefficients}

The heat kernel $U_{E}(t, x, y)$ is defined as the solution of the heat equation

$\frac{\partial U_{E}(t, x, y)}{\partial t}=\left(\nabla^{2}+E\right) U_{E}(t, x, y)$,

subject to the initial condition

$U_{E}(0, x, y)=\frac{\delta(x-y)}{\sqrt{g}}$.

Note that the kernel $U_{E}(t, x, y)$ may contain Lorentz indices if we consider vector or tensor degrees of freedom. Throughout this section these indices are suppressed. By definition, $U_{E}(t, x, y)$ has the dimension of an inverse volume. Also, $t$ is the proper time parameter with mass dimension two, $-\nabla^{2}$ is the Laplacian, and $E$ an endomorphism. The formal solution of (1) is given by

$U_{E}(t, x, y)=e^{t\left(\nabla^{2}+E\right)}$.

Due to the symmetries of the chosen background, $U_{E}(t, x, y)$ can only depend on the proper time $t$ and the distance between the points $x$ and $y$. Therefore, defining $\sigma$ to be half the square of the geodesic distance between $x$ and $y$, we may write

$U_{E}(t, x, y)=U_{E}(t, \sigma)$.

For early times we expand the heat kernel as an asymptotic series following the DeWitt ansatz

$$
\begin{aligned}
U_{E}(t, \sigma)= & \frac{\Delta^{1 / 2}}{(4 \pi t)^{d / 2}} \exp \left\{-\frac{\sigma}{2 t}\right\} \sum_{n=0}^{\infty}\left[\tilde{b}_{2 n}(E, \sigma) t^{n}\right. \\
& \left.+\tilde{c}_{d+2 n}(E, \sigma) t^{d / 2+n}\right],
\end{aligned}
$$

where $\Delta$ is the Van Fleck-Morette determinant

$\Delta=\frac{\operatorname{det}\left[-\nabla_{\mu}^{x} \nabla_{\nu}^{y} \sigma(x, y)\right]}{g^{1 / 2}(x) g^{1 / 2}(y)}$.

By definition, the heat kernel coefficients $\tilde{b}_{m}$ and $\tilde{c}_{m}$ have canonical mass dimension $m$ in any dimension. Just as $U_{E}$, these coefficients may carry Lorentz indices which are suppressed here.

Note that the ansatz (5) seems slightly different from those used in the literature $[9,42,48,49]$ through the appearance of 
the coefficients $\tilde{c}_{d+2 n}$. These terms only arise for heat kernels of constrained fields, such as transverse vector and transverse traceless tensor fields, and are related to the exclusion of lowest modes [11,50-52]. Excluded modes always produce contributions of the form $\exp (\alpha R t)$ which invariably give rise to terms with positive integer powers of the proper-time parameter when expanded for small times. For even dimensions, the terms $\tilde{c}_{d+2 n}$ could be combined with the $\tilde{b}_{d+2 n}$ coefficients of the same mass dimension. In odd dimensions, however, all $\tilde{c}_{d+2 n}$ coefficients have mass dimensions different from all $\tilde{b}_{2 n}$ coefficients, and cannot be combined into a single coefficient. Hence, for the sake of generality, and given their distinctly different origins, we keep these coefficients separate for now. The coefficients $\tilde{c}_{d+2 n}$ vanish for unconstrained fields as we will show a posteriori (see Sect. 3.5).

Since we are ultimately interested in the trace of the heat kernel, we only need the coincidence limit of $U_{E}(t, \sigma)$. Using (5), we define the coincidence limit of the heat kernel coefficients, $\tilde{b}_{2 n}(E)$ and $\tilde{c}_{d+2 n}(E)$, for given endomorphism $E$ as

$\tilde{b}_{2 n}(E)=\tilde{b}_{2 n}(E, 0), \quad \tilde{c}_{d+2 n}(E)=\tilde{c}_{d+2 n}(E, 0)$.

Then, the trace of the heat kernel is given by

$$
\begin{aligned}
\operatorname{Tr}_{s} U_{E}(t, \sigma)= & \frac{1}{(4 \pi t)^{d / 2}} \sum_{n=0}^{\infty}\left[\operatorname{Tr}_{s}\left[\tilde{b}_{2 n}(E)\right] t^{n}\right. \\
& \left.+\operatorname{Tr}_{s}\left[\tilde{c}_{d+2 n}(E)\right] t^{d / 2+n}\right],
\end{aligned}
$$

where the trace acts on the coordinate dependence as well as any Lorentz indices carried by $\tilde{b}_{2 n}$ and $\tilde{c}_{d+2 n}$. Further, the index $s$ denotes the spin of the field w.r.t. which the trace is acting on. Since the heat kernel coefficients are coordinate independent on a fully symmetric background, we may define

$b_{n}^{(s)}(E)=\frac{1}{\operatorname{Vol}} \operatorname{Tr}_{s}\left[\tilde{b}_{n}(E)\right], \quad c_{n}^{(s)}(E)=\frac{1}{\operatorname{Vol}} \operatorname{Tr}_{s}\left[\tilde{c}_{n}(E)\right]$,

in which the volume of the $d$-dimensional sphere is

$\mathrm{Vol}=\frac{2 \pi^{(d+1) / 2}}{\Gamma\left(\frac{d+1}{2}\right)}\left(\frac{d(d-1)}{R}\right)^{d / 2}$

and $R$ denotes the Ricci scalar curvature. This allows us to write

$$
\begin{aligned}
& \operatorname{Tr}_{s} U_{E}(t, \sigma) \\
& =\frac{\mathrm{Vol}}{(4 \pi t)^{d / 2}} \sum_{n=0}^{\infty}\left[b_{2 n}^{(s)}(E) t^{n}+c_{d+2 n}^{(s)}(E) t^{d / 2+n}\right] .
\end{aligned}
$$

Finally, we notice that the heat kernel for a given endomorphism $\bar{E}$ is related to that for any other endomorphism $E$ by

$U_{E}(t, \sigma)=e^{t(E-\bar{E})} U_{\bar{E}}(t, \sigma)$, assuming that the endomorphism commutes with the covariant derivative on the sphere. This relation implies that the corresponding heat kernel coefficients are related by

$$
\begin{aligned}
b_{2 n}^{(s)}(E) & =\sum_{k=0}^{n} \frac{(E-\bar{E})^{k}}{k !} b_{2(n-k)}^{(s)}(\bar{E}), \\
c_{d+2 n}^{(s)}(E) & =\sum_{k=0}^{n} \frac{(E-\bar{E})^{k}}{k !} c_{d+2(n-k)}^{(s)}(\bar{E}),
\end{aligned}
$$

which serves as a definition of heat kernel coefficients for arbitrary endomorphisms.

\subsection{Renormalisation group}

An important area for the application of heat kernels is Wilson's (functional) renormalisation group. The technique amounts to the introduction of an infrared momentum cutoff $k$ into the path integral definition of quantum or statistical field theory, which induces a scale-dependence $k \partial_{k}$ in the form of an exact functional flow for the effective action $\Gamma_{k}$ (see [53-55] for reviews),

$k \partial_{k} \Gamma_{k}=\frac{1}{2} \operatorname{Tr}\left\{\left(k \partial_{k} \mathcal{R}_{k}\right)\left(\Gamma_{k}^{(2)}+\mathcal{R}_{k}\right)^{-1}\right\}$.

Here, $\Gamma_{k}^{(2)}$ denotes the second variation of $\Gamma_{k}$. The function $\mathcal{R}_{k}$ denotes the Wilsonian IR regulator, chosen such that $\Gamma_{k}$ interpolates between the microscopic theory $(1 / k \rightarrow 0)$ and the full quantum effective action $(k \rightarrow 0)$, see [44]. At weak coupling, iterative solutions generate perturbation theory to all loop orders [56,57]. At strong coupling, nonperturbative approximations such as the derivative expansion, vertex expansions, or mixtures thereof are available $[53,58,59]$. The stability and convergence of approximations can be controlled as well [60-62].

Our main point here relates to the operator trace in (14), which for many applications can be evaluated on flat Euclidean backgrounds. For quantum field theories on curved backgrounds, or for studies of fully-fledged quantum gravity, it is often convenient to evaluate the operator trace on suitably chosen non-flat backgrounds [29,31,63,64] also using the background field method [65] and optimised cutoffs [32,44,66,67]. In quantum gravity, this has enabled advanced tests of the asymptotic safety conjecture on spheres $[32,33,35,40,41,43,68]$. Further applications of heat kernels include flow equations on Einstein [69] or hyperbolic spaces [70], critical fields on curved backgrounds [71], low energy effective actions [72], and proper-time flows [73].

To see how heat kernel coefficients enter in this methodology we note that typical contributions on the right-hand 
side of (14) are given by traces of operators in the form $\left(k \partial_{k} \mathcal{R}_{k}\right)\left(\Gamma_{k}^{(2)}+\mathcal{R}_{k}\right)^{-1}$. After inserting the second variation matrix and choosing a regulator the integrand can be represented as a (matrix-valued) function $W\left(-\nabla^{2}\right)$ of the Laplacian. Using the Laplace anti-transformation

$W(z)=\int_{0}^{\infty} \mathrm{d} t \widetilde{W}(t) e^{-t z}$

we may then express the desired trace as

$\operatorname{Tr} W\left(-\nabla^{2}\right)=\int_{0}^{\infty} \mathrm{d} t \tilde{W}(t) \operatorname{Tr} e^{t \nabla^{2}}$

Crucially, the trace $\operatorname{Tr} e^{t \nabla^{2}}$ appearing on the right-hand side is the trace of the heat kernel with vanishing endomorphism (3). Thus, the early time expansion (5) allows us to evaluate the trace (15) in terms of the heat kernel coefficients $b_{2 n}$ and $c_{d+2 n}$ via

$$
\begin{aligned}
\operatorname{Tr} W\left(-\nabla^{2}\right)= & \frac{\operatorname{Vol}}{(4 \pi)^{d / 2}} \sum_{n=0}^{\infty}\left[b_{2 n}(0) \int_{0}^{\infty} \mathrm{d} t t^{n-d / 2} \widetilde{W}(t)\right. \\
& \left.+c_{d+2 n}(0) \int_{0}^{\infty} \mathrm{d} t t^{n} \widetilde{W}(t)\right] .
\end{aligned}
$$

We conclude that the heat kernel coefficients $b_{2 n}(0)$ and $c_{d+2 n}(0)$ are key inputs for Wilsonian flows on maximally symmetric backgrounds. The calculation of all heat kernel coefficients $b_{2 n}$ and $c_{d+2 n}(n \geq 0)$ on spheres in arbitrary dimension is the topic of the following sections.

\section{Heat kernels from Green's functions}

In this section, we find Green's functions for scalars, transverse vectors, and transverse traceless tensors and use these to extract heat kernel coefficients for Laplacians on spheres in any dimension. We also give results for heat kernels of unconstrained vectors and tensors.

\subsection{Green's function technique}

Our methodology largely follows Avramidi [9] and starts by noting that the heat kernel defined in (5) can be connected to a Green's function using the Schwinger-DeWitt representation

$G(\sigma)=\int_{0}^{\infty} \mathrm{d} t e^{-t m^{2}} U_{E}(t, \sigma)$.

By definition, $G(\sigma)$ has canonical mass dimension $M^{d-2}$. Using (1) with (2) it is straightforward to show that $G(\sigma)$ in (17) is a Green's function for the differential operator $\left(-\nabla^{2}+\right.$ $\left.m^{2}-E\right)$. In the coincidence limit $\sigma=0$, we can write

$$
\begin{aligned}
\frac{\operatorname{Tr}_{s} G(\sigma)}{\operatorname{Vol}}= & \frac{1}{(4 \pi)^{d / 2}} \int_{0}^{\infty} \mathrm{d} t e^{-t m^{2}} \sum_{n=0}^{\infty}\left[b_{2 n}^{(s)}(E) t^{-d / 2+n}\right. \\
& \left.+c_{d+2 n}^{(s)}(E) t^{n}\right]
\end{aligned}
$$

Note that the trace on the left-hand side effectively only takes the trace of the tensor structure of $G(\sigma)$, the trace over the coordinate dependence drops out due to the volume factor in the denominator. Recalling the elementary definition of the $\Gamma$-function, $\Gamma(n+1)=\int_{0}^{\infty} \mathrm{d} s s^{n} e^{-s}$, and substituting $s=t m^{2}$, the $t$-integration in (18) is performed term by term. Doing so, the Green's function takes the form of a large- $m$ expansion

$$
\begin{aligned}
\frac{\operatorname{Tr}_{s} G(\sigma)}{\operatorname{Vol}}= & \frac{m^{d-2}}{(4 \pi)^{d / 2}} \sum_{n=0}^{\infty}\left[\Gamma\left(n-\frac{d}{2}+1\right) \frac{b_{2 n}^{(s)}(E)}{m^{2 n}}\right. \\
& \left.+\Gamma(n+1) \frac{c_{d+2 n}^{(s)}(E)}{m^{d+2 n}}\right] .
\end{aligned}
$$

Hence, by calculating the large- $m$ expansion of the Green's function at its coincidence limit, we may read off the heat kernel coefficients as the corresponding Taylor coefficients. This fact is exploited below to calculate all heat kernel coefficients for scalars, transverse vectors, and transverse traceless symmetric tensors.

\subsection{Scalars}

We begin with the Green's function for scalar fields $G_{Q}(\sigma)$ to explain how the corresponding heat kernel coefficients are computed in practice. This follows closely the derivation given in [9]. The Green's function for scalar fields is the solution of the differential equation

$\left(-\nabla^{2}+Q\right) G_{Q}(\sigma)=\frac{1}{\sqrt{g}} \delta(x-y)$.

In $[45,74,75]$ it has been explained why solutions can be expressed in terms of a hypergeometric function,

$G_{Q}(\sigma)=\frac{\Gamma(a) \Gamma(b)}{\Gamma(d / 2)} \frac{r^{2-d}}{(4 \pi)^{d / 2}}{ }_{2} F_{1}(a, b ; c ; z)$

with parameters

$$
\begin{aligned}
& a=\frac{d-1}{2}+\xi, \quad b=\frac{d-1}{2}-\xi, \quad c=\frac{d}{2}, \\
& \xi=\sqrt{\frac{(d-1)^{2}}{4}-Q r^{2},}
\end{aligned}
$$


and

$z=\cos ^{2}\left(\sqrt{\frac{\sigma}{2 r^{2}}}\right)$.

In the latter, $r$ denotes the radius of the sphere which relates to the Ricci scalar curvature $R$ as

$\frac{R}{d(d-1)}=r^{-2}$

To find the coincidence limit for (21), we follow Avramidi [9] and exploit a useful representation for the hypergeometric function [76]

${ }_{2} F_{1}(a, b ; c ; 1)=\frac{\Gamma(c) \Gamma(c-a-b)}{\Gamma(c-a) \Gamma(c-b)}$

to arrive at

$$
\begin{aligned}
G_{Q}(0)= & \frac{r^{2-d}}{(4 \pi)^{d / 2}} \Gamma\left(1-\frac{d}{2}\right) \\
& \times \frac{\Gamma\left(\frac{d-1}{2}+\xi\right) \Gamma\left(\frac{d-1}{2}-\xi\right)}{\Gamma\left(\frac{1}{2}+\xi\right) \Gamma\left(\frac{1}{2}-\xi\right)} .
\end{aligned}
$$

The large mass expansion requires an expansion of the Gamma functions which can be done noting that [77]

$$
\begin{aligned}
\ln [\Gamma(\alpha+\xi)]= & \left(\alpha+\xi-\frac{1}{2}\right) \ln (\xi)-\xi+\frac{1}{2} \ln (2 \pi) \\
& +\sum_{n=2}^{\infty} \frac{(-1)^{n} B_{n}(\alpha)}{n(n-1) \xi^{n-1}}
\end{aligned}
$$

with $|\xi| \rightarrow \infty,|\operatorname{ph}(\xi)|<\pi$, and $B_{n}(x)$ being the Bernoulli polynomials. Then, using $B_{n}\left(\frac{1}{2}\right)=0$ for $n=$ odd, we find

$$
\frac{\Gamma\left(\frac{d-1}{2}+\xi\right) \Gamma\left(\frac{d-1}{2}-\xi\right)}{\Gamma\left(\frac{1}{2}+\xi\right) \Gamma\left(\frac{1}{2}-\xi\right)}=\sum_{n=0}^{\infty} \kappa_{n}(d)\left(-\xi^{2}\right)^{d / 2-1-n}
$$

with the generating function for the coefficients $\kappa_{n}(d)$ given by

$$
\exp \sum_{n=1}^{\infty} \frac{(-1)^{n+1}}{n(2 n+1)} B_{2 n+1}\left(\frac{d-1}{2}\right) z^{n}=\sum_{n=0}^{\infty} \kappa_{n}(d) z^{n} .
$$

Note that the expression (26) is only valid for $\Im(\xi) \neq 0$. To proceed, we split $Q$ into a mass part $m^{2}$ and an endomorphism part $E$ through $Q=m^{2}-E$. The endomorphism part $E$ can be chosen such that $\xi$ becomes proportional to the mass $m$. Using (22) with (24), this requirement uniquely fixes the endomorphism $E=\bar{E}$ and $\xi$ to

$$
\begin{aligned}
\bar{E} & =\frac{1-d}{4 d} R, \\
-\xi^{2} & =d(d-1) \frac{m^{2}}{R},
\end{aligned}
$$

and we get

$\left.G_{Q}(0)\right|_{\bar{E}}=\frac{1}{(4 \pi)^{d / 2}} \Gamma\left(1-\frac{d}{2}\right) \sum_{n=0}^{\infty} \kappa_{n}(d) r^{-2 n} m^{d-2-2 n}$.

For the endomorphism (28) we can now read off the heat kernel coefficients $b_{2 n}^{(0)}$ and $c_{d+2 n}^{(0)}$ by comparison with (19). To distinguish them, we note that the coefficients $c_{d+2 n}^{(0)}$ enter with $d$-independent integer powers of $m$ in (19) while the coefficients $b_{2 n}^{(0)}$ have $d$-dependent powers of $m$. Moreover, the coefficients $b_{2 n}^{(0)}\left(c_{d+2 n}^{(0)}\right)$ are linear in (independent of) the parameters $\kappa_{i}(d)$. This is because the $d$-dependent powers of $m$ originate from (26) which is linear in the $\kappa_{i}(d)$. Exploiting this fact, we get from (29)

$$
\begin{aligned}
b_{2 n}^{(0)}(\bar{E}) & =\frac{\Gamma\left(1-\frac{d}{2}\right)}{\Gamma\left(1+n-\frac{d}{2}\right)}\left(\frac{R}{d(d-1)}\right)^{n} \kappa_{n}(d), \\
c_{d+2 n}^{(0)}(\bar{E}) & =0,
\end{aligned}
$$

with $\kappa_{n}(d)$ determined through (27). Moreover, with the help of (13), we find the heat kernel coefficients for scalar fields and arbitrary endomorphism $E$,

$$
\begin{aligned}
b_{2 n}^{(0)}(E)= & \sum_{k=0}^{n} \frac{\Gamma\left(1-\frac{d}{2}\right)}{k ! \Gamma\left(1+n-k-\frac{d}{2}\right)}\left(\frac{(d-1) R}{4 d}+E\right)^{k} \\
& \times\left(\frac{R}{(d-1) d}\right)^{n-k} \kappa_{n-k}(d), \\
c_{d+2 n}^{(0)}(E)= & 0 .
\end{aligned}
$$

Table 1 summarises our results for the first few scalar heat kernel coefficients (31) for $E=0$ and a selection of integer dimensions.

Finally, we note that the heat kernel expansion with (31) is asymptotic in even dimensions but has a finite radius of convergence in odd ones, owing to the propagator (25) being non-finite only in even dimensions [9]. The result generalises to non-integer dimensions.

\subsection{Transverse vectors}

Next, we determine the heat kernel coefficients for transverse vector fields. To that end, we review the derivation of the transverse vector Green's function, closely following 
Table 1 The scalar heat kernel coefficients for different integer dimensions and vanishing endomorphism

\begin{tabular}{llllll}
\hline & $d=2$ & $d=3$ & $d=4$ & $d=5$ & $d=6$ \\
\hline$b_{0}^{(0)}$ & 1 & 1 & 1 & 1 & 1 \\
$b_{2}^{(0)}$ & $\frac{1}{6} R$ & $\frac{1}{6} R$ & $\frac{1}{6} R$ & $\frac{1}{6} R$ & $\frac{1}{6} R$ \\
$b_{4}^{(0)}$ & $\frac{1}{60} R^{2}$ & $\frac{1}{72} R^{2}$ & $\frac{29}{2160} R^{2}$ & $\frac{1}{75} R^{2}$ & $\frac{1}{75} R^{2}$ \\
$b_{6}^{(0)}$ & $\frac{1}{630} R^{3}$ & $\frac{1}{1296} R^{3}$ & $\frac{37}{54432} R^{3}$ & $\frac{1}{1500} R^{3}$ & $\frac{1}{17139}$ \\
$b_{8}^{(0)}$ & $\frac{1}{5040} R^{4}$ & $\frac{1}{31104} R^{4}$ & $\frac{149}{6531840} R^{4}$ & $\frac{1}{45000} R^{4}$ & $\frac{833}{3650000} R^{4}$ \\
$b_{10}^{(0)}$ & $\frac{1}{27720} R^{5}$ & $\frac{1}{933120} R^{5}$ & $\frac{119}{431101440} R^{5}$ & $\frac{1}{2250000} R^{5}$ & $\frac{137}{267300000} R^{5}$ \\
\hline
\end{tabular}

$[46,75]$. We then exploit the result to derive all heat kernel coefficients for transverse vector fields on spheres.

It is important to notice that the Green's function for transverse vector fields $G_{Q, \mu \nu^{\prime}}^{T}(\sigma)$ fulfils a differential equation of the form

$\left(-\nabla^{2}+Q\right) G_{Q, \mu \nu^{\prime}}^{T}(\sigma)=\frac{g_{\mu \nu^{\prime}}}{\sqrt{g}} \delta(x-y)+$ longitudinal terms.

The longitudinal terms ensure the transversality of the righthand side. They can be derived by considering the full vector Green's function and splitting it into a transverse and a longitudinal part. As pointed out in [46], neglecting these longitudinal terms can lead to inconsistent results for the Green's function.

To solve the differential equation for the transverse vector Green's function, we want to reduce it to a scalar function $S^{T}(\sigma)$, which is called the structure function. For this, the transverse vector projector

$\mathcal{P}_{\mu}{ }^{v}=g_{\mu}{ }^{\nu} \nabla^{2}-\nabla^{v} \nabla_{\mu}$

is introduced. It fulfils the properties $[46,75]$

$$
\begin{aligned}
\nabla^{\mu} \mathcal{P}_{\mu}{ }^{\nu} T_{\nu} & =\mathcal{P}_{\nu}{ }^{\mu}\left(\nabla_{\mu} S(\sigma)\right)=0, \\
{\left[\nabla^{2}, \mathcal{P}_{\mu}{ }^{\nu}\right] T_{\nu} } & =0, \\
\mathcal{P}_{\mu}{ }^{\nu} \mathcal{P}_{\nu}{ }^{\rho} T_{\rho} & =\mathcal{P}_{\mu}{ }^{\rho}\left(\nabla^{2}-\frac{R}{d}\right) T_{\rho},
\end{aligned}
$$

for an arbitrary vector $T_{\mu}$. From this, it follow that acting with $\mathcal{P}_{\mu}{ }^{\alpha} \mathcal{P}_{\nu^{\prime}}{ }^{\beta^{\prime}}$ on any bi-tensor $T_{\alpha \beta^{\prime}}$ gives rise to a bi-tensor which is transverse in both indices. Hence, it has the right properties to be the Green's function for transverse vector fields. With this in mind, we make an ansatz for the transverse vector Green's function through

$G_{Q, \mu \nu^{\prime}}^{T}(\sigma)=\mathcal{P}_{\mu}{ }^{\alpha} \mathcal{P}_{\nu^{\prime}}^{\beta^{\prime}}\left(\mathcal{R}_{\alpha \beta^{\prime}} S^{T}(\sigma)\right)$.

The bi-tensor $\mathcal{R}_{\alpha \beta^{\prime}}$ is arbitrary and can be chosen to simplify the computations. One choice is given by $[46,75]$

$$
\begin{aligned}
\mathcal{R}_{\alpha \beta^{\prime}} & =-2 r^{2} \nabla_{\alpha} \nabla_{\beta^{\prime}} \sin ^{2}\left(\frac{\sqrt{2 \sigma}}{2 r}\right) \\
& =g_{\alpha \beta^{\prime}}+\frac{\sigma_{\alpha} \sigma_{\beta^{\prime}}}{\sigma} \sin ^{2}\left(\frac{\sqrt{2 \sigma}}{2 r}\right),
\end{aligned}
$$

with $\sigma_{\alpha}=\nabla_{\alpha} \sigma$. The right-hand side of this equation can be derived using the relations in (43) and (44). It fulfils the property

$$
\begin{aligned}
\nabla^{2}\left(\mathcal{R}_{\alpha \beta^{\prime}} S(\sigma)\right)= & \mathcal{R}_{\alpha \beta^{\prime}}\left(\nabla^{2}+\frac{R}{d(d-1)}\right) S(\sigma) \\
& + \text { longitudinal, }
\end{aligned}
$$

with $S(\sigma)$ being an arbitrary scalar. Since this bi-tensor is always contracted with transverse vector projectors, the longitudinal terms can be neglected. Using the above structures and identities, and contracting (32) with $\mathcal{P}_{\rho}{ }^{\mu} \mathcal{P}_{\sigma^{\prime}} v^{\prime}$ we arrive at

$$
\begin{aligned}
& \mathcal{P}_{\rho}{ }_{\rho}^{\alpha} \mathcal{P}_{\sigma^{\prime}}{ }^{\beta^{\prime}}\left[\mathcal{R}_{\alpha \beta^{\prime}}\left(-\nabla^{2}+Q-\frac{R}{d(d-1)}\right)\right. \\
& \left.\times\left(\nabla^{2}+\frac{2-d}{d(d-1)} R\right)^{2} S^{T}(\sigma)\right] \\
& =\mathcal{P}_{\rho}{ }^{\alpha} \mathcal{P}_{\sigma^{\prime}}{ }^{\beta^{\prime}}\left[\frac{\mathcal{R}_{\alpha \beta^{\prime}}}{\sqrt{g}} \delta(x-y)\right] .
\end{aligned}
$$

We observe that the structure function for the transverse vector Green's function obeys the differential equation

$$
\begin{aligned}
& \left(-\nabla^{2}+Q-\frac{R}{d(d-1)}\right)\left(\nabla^{2}+\frac{2-d}{d(d-1)} R\right)^{2} S^{T}(\sigma) \\
& =\frac{1}{\sqrt{g}} \delta(x-y)
\end{aligned}
$$

which can be solved with the help of the scalar Green's function $[46,75]$. Its solution takes the explicit form

$S^{T}(\sigma)=G_{\chi_{1}, \chi_{2}, \chi_{2}}(\sigma) \equiv-\frac{\partial}{\partial \chi_{2}} G_{\chi_{1}, \chi_{2}}(\sigma)$, 
where

$G_{\chi_{1}, \chi_{2}}(\sigma)=\frac{G_{\chi_{1}}(\sigma)-G_{\chi_{2}}(\sigma)}{\chi_{2}-\chi_{1}}$,

and $G_{\chi}(\sigma)$ given in (21), and all of this evaluated at

$\chi_{1}=Q-\frac{R}{d(d-1)}, \quad \chi_{2}=\frac{d-2}{d(d-1)} R$.

Using the result for the structure function, the transverse vector Green's function is now given by

$G_{Q, \mu \nu^{\prime}}^{T}(\sigma)=\mathcal{P}_{\mu}^{\alpha} \mathcal{P}_{\nu^{\prime}}^{\beta^{\prime}}\left(\mathcal{R}_{\alpha \beta^{\prime}} G_{\chi_{1}, \chi_{2}, \chi_{2}}(\sigma)\right)$.

To extract the heat kernel coefficients we need to contract this with $g^{\mu v^{\prime}}$ and calculate the coincidence limit of the resulting expression. Covariant derivatives acting on $\sigma_{\alpha}$ can be computed using [47]

$$
\begin{aligned}
\nabla_{\nu^{\prime}} \sigma_{\mu} & =C(\sigma)\left[g_{\mu \nu^{\prime}}+\frac{1}{2 \sigma} \sigma_{\mu} \sigma_{v^{\prime}}\right]+\frac{1}{2 \sigma} \sigma_{\mu} \sigma_{v^{\prime}}, \\
\nabla_{\nu} \sigma_{\mu} & =A(\sigma)\left[g_{\mu \nu}-\frac{1}{2 \sigma} \sigma_{\mu} \sigma_{\nu}\right]+\frac{1}{2 \sigma} \sigma_{\mu} \sigma_{\nu}, \\
\nabla_{\mu} g_{\alpha \beta^{\prime}} & =-\frac{A(\sigma)+C(\sigma)}{2 \sigma}\left(g_{\mu \alpha} \sigma_{\beta^{\prime}}+g_{\mu \beta^{\prime}} \sigma_{\alpha}\right),
\end{aligned}
$$

with

$$
\begin{aligned}
& A(\sigma)=\sqrt{\frac{2 \sigma R}{d(d-1)}} \cot \left(\sqrt{\frac{2 \sigma R}{d(d-1)}}\right), \\
& C(\sigma)=-\sqrt{\frac{2 \sigma R}{d(d-1)}} \csc \left(\sqrt{\frac{2 \sigma R}{d(d-1)}}\right) .
\end{aligned}
$$

Acting with the transverse vector projectors can be simplified by performing a series expansion of $\mathcal{R}_{\alpha \beta^{\prime}} G_{\chi_{1} \chi_{2}}(\sigma)$ in the coincidence limit. Observing that $\sigma_{\mu} \sigma^{\mu}=2 \sigma$ and that $\sigma_{\mu}$ does not contribute in the coincidence limit, we may employ $\sigma_{\mu}$ as an expansion parameter. Coming from the transverse vector projectors, we have four covariant derivatives acting on this expression. Hence, an expansion up to order four in $\sigma_{\mu}$ is needed. Expanding the structure function as

$S^{T}(\sigma)=\sum_{n=0}^{\infty} S_{n}^{T} \sigma^{n}$

the expansion of $\mathcal{R}_{\alpha \beta^{\prime}} G_{\chi_{1}, \chi_{2}}(\sigma)$ gives

$$
\begin{aligned}
\mathcal{R}_{\alpha \beta^{\prime}} G_{\chi_{1}, \chi_{2}}(\sigma) \\
=S_{0}^{T}\left(g_{\alpha \beta^{\prime}}+\frac{R}{2 d(d-1)} \sigma_{\alpha} \sigma_{\beta^{\prime}}-\frac{\sigma R^{2}}{12 d^{2}(d-1)^{2}} \sigma_{\alpha} \sigma_{\beta^{\prime}}\right) \\
\quad+S_{1}^{T}\left(\sigma g_{\alpha \beta^{\prime}}+\frac{\sigma R}{2 d(d-1)} \sigma_{\alpha} \sigma_{\beta^{\prime}}\right)+S_{2}^{T} \sigma^{2} g_{\alpha \beta^{\prime}} \\
\quad+\mathcal{O}\left(\sigma_{\mu}\right)^{5} .
\end{aligned}
$$

After acting with the projectors and contracting with $g^{\mu v^{\prime}}$, we find

$g^{\mu v^{\prime}} G_{Q, \mu v^{\prime}}^{T}(0)=\frac{8-5 d}{3} R S_{1}^{T}+2 d\left(d^{2}+d-2\right) S_{2}^{T}$.

Explicit expressions for the expansion coefficients $S_{1}^{T}$ and $S_{2}^{T}$ can be found in Appendix A. Inserting the expressions for $S_{1}^{T}$ and $S_{2}^{T}$ and using (26), we find

$$
\begin{aligned}
g^{\mu \nu^{\prime}} G_{Q, \mu \nu^{\prime}}^{T}(0) & \\
= & \frac{1}{(4 \pi)^{d / 2}}\left(\frac{d(d-1)}{R}\right)^{1-d / 2}\left[\frac{R \Gamma(d-1)}{\Gamma\left(\frac{d}{2}\right)(d Q-R)}\right. \\
& +\frac{\pi\left(d^{2} Q-d Q-R\right)}{\sin \left(\frac{\pi d}{2}\right) \Gamma\left(\frac{d}{2}\right)(d Q-R)} \\
& \left.\times \sum_{k=0}^{\infty} \kappa_{k}(d)\left(-\bar{\xi}^{2}\right)^{d / 2-k-1}\right],
\end{aligned}
$$

with

$\bar{\xi}=\frac{1}{2} \sqrt{d\left(-\frac{4(d-1) Q}{R}+d-2\right)+5 .}$

To expand (48) in the large $m$ limit, we use the same trick as in the scalar case and set $Q=m^{2}-E$ for an endomorphism such that $\bar{\xi}$ is directly proportional to $m$. We find

$$
\begin{aligned}
\bar{E} & =\frac{-5+2 d-d^{2}}{4 d(d-1)} R, \\
-\bar{\xi}^{2} & =d(d-1) \frac{m^{2}}{R} .
\end{aligned}
$$

Using (50) and the geometric series to expand denominators containing $m$ in the large $m$ limit, we arrive at 
Table 2 The vector heat kernel coefficients for different integer dimensions and vanishing endomorphism

\begin{tabular}{llllll}
\hline & $d=2$ & $d=3$ & $d=4$ & $d=5$ & $d=6$ \\
\hline$b_{0}^{(1)}$ & 1 & 2 & 3 & 4 & 5 \\
$c_{d}^{(1)}$ & $\frac{1}{2} R$ & $\frac{2}{3 \sqrt{6 \pi}} R^{3 / 2}$ & $\frac{1}{24} R^{2}$ & $\frac{1}{25 \sqrt{5 \pi}} R^{5 / 2}$ & $\frac{1}{450} R^{3}$ \\
$b_{2}^{(1)}$ & $-\frac{1}{3} R$ & 0 & $\frac{1}{4} R$ & $\frac{7}{15} R$ & $\frac{2}{3} R$ \\
$c_{d+2}^{(1)}$ & $\frac{1}{4} R^{2}$ & $\frac{2}{9 \sqrt{6 \pi}} R^{5 / 2}$ & $\frac{1}{96} R^{3}$ & $\frac{1}{125 \sqrt{5 \pi}} R^{7 / 2}$ & $\frac{1}{2700} R^{4}$ \\
$b_{4}^{(1)}$ & $-\frac{11}{40} R^{2}$ & $-\frac{1}{9} R^{2}$ & $-\frac{67}{1440} R^{2}$ & $-\frac{1}{120} R^{2}$ & $\frac{1}{360} R^{9 / 2}$ \\
$c_{d+4}^{(1)}$ & $\frac{1}{16} R^{3}$ & $\frac{1}{27 \sqrt{6 \pi}} R^{7 / 2}$ & $\frac{1}{768} R^{4}$ & $-\frac{1}{160} R^{3}$ & $\frac{1}{32400} R^{5}$ \\
$b_{6}^{(1)}$ & $-\frac{37}{504} R^{3}$ & $-\frac{2}{81} R^{3}$ & $-\frac{4321}{362880} R^{3}$ & $\frac{1}{18750 \sqrt{5 \pi}} R^{11 / 2}$ & $-\frac{2539}{850500} R^{3}$ \\
$c_{d+6}^{(1)}$ & $\frac{1}{96} R^{4}$ & $\frac{1}{243 \sqrt{6 \pi}} R^{9 / 2}$ & $\frac{1}{9216} R^{5}$ & $-\frac{17}{23040} R^{4}$ & $\frac{1}{583200} R^{6}$ \\
$b_{8}^{(1)}$ & $-\frac{157}{13440} R^{4}$ & $-\frac{1}{324} R^{4}$ & $-\frac{3397}{2488320} R^{4}$ & $\frac{1}{375000 \sqrt{5 \pi}} R^{13 / 2}$ & $-\frac{176107}{408240000} R^{4}$ \\
$c_{d+8}^{(1)}$ & $\frac{1}{768} R^{5}$ & $\frac{1}{2916 \sqrt{6 \pi}} R^{11 / 2}$ & $\frac{1}{147456} R^{6}$ & 1 \\
$b_{10}^{(1)}$ & $-\frac{109}{88704} R^{5}$ & $-\frac{1}{3645} R^{5}$ & $-\frac{245461}{2299207680} R^{5}$ & $-\frac{1}{18432} R^{5}$ & $\frac{1}{2996800} R^{7}$ \\
$c_{d+10}^{(1)}$ & $\frac{1}{7680} R^{6}$ & $\frac{1}{43740 \sqrt{6 \pi}} R^{13 / 2}$ & $\frac{1}{2949120} R^{7}$ & $\frac{213749}{6735960000} R^{5}$ \\
\hline
\end{tabular}

$$
\begin{aligned}
& \left.g^{\mu v^{\prime}} G_{Q, \mu v^{\prime}}^{T}(0)\right|_{\bar{E}} \\
& =\frac{(d-1)^{2}}{(4 \pi)^{d / 2}} \frac{\pi}{\sin (d \pi / 2) \Gamma(d / 2)}\left(\frac{1}{d-1}+\frac{R}{4 d m^{2}}\right) \\
& \quad \times\left(m^{2}\right)^{d / 2-1} \sum_{k=0}^{\infty}\left(\frac{R}{m^{2}}\right)^{k} \sum_{\ell=0}^{k}\left(-\frac{(d-3)^{2}}{4}\right)^{\ell} \\
& \quad \times \frac{\kappa_{k-\ell}(d)}{(d(d-1))^{k}}+\frac{R \Gamma(d-1)}{d m^{2}(4 \pi)^{d / 2}} \frac{\left(\frac{d(d-1)}{R}\right)^{1-d / 2}}{\Gamma(d / 2)} \\
& \quad \times \sum_{k=0}^{\infty}\left(-\frac{(d-3)^{2}}{4 d(d-1)} \frac{R}{m^{2}}\right)^{k},
\end{aligned}
$$

where we have reorganised sums coming from geometric series and (26) to combine powers of $m$. As before, the transverse vector heat kernels $b_{2 n}^{(1)}$ and $c_{d+2 n}^{(1)}$ for the endomorphism (50) can now be read off from (51) by noticing that the coefficients $b_{2 n}^{(1)}\left(c_{d+2 n}^{(1)}\right)$ are linear in (independent of) the parameters $\kappa_{i}(d)$. We find

$$
\begin{aligned}
b_{2 n}^{(1)}(\bar{E})=\frac{(d-1) \pi R^{n}}{\Gamma(1+n-d / 2)}\left[\sum_{\ell=0}^{n}\left(-\frac{(d-3)^{2}}{4}\right)^{\ell}\right. \\
\quad \times \frac{\kappa_{n-\ell}(d)(d(d-1))^{-n}}{\sin (d \pi / 2) \Gamma(d / 2)} \\
+\frac{(d-1)^{2}}{4} \sum_{\ell=0}^{n-1}\left(-\frac{(d-3)^{2}}{4}\right)^{\ell} \\
\left.\times \frac{\kappa_{n-1-\ell}(d)(d(d-1))^{-n}}{\sin (d \pi / 2) \Gamma(d / 2)}\right]
\end{aligned}
$$

$$
\begin{gathered}
c_{d+2 n}^{(1)}(\bar{E})=\frac{\Gamma(d)}{\Gamma(d / 2) \Gamma(1+n)}\left(\frac{R}{d(d-1)}\right)^{d / 2} \\
\times\left(-\frac{(d-3)^{2}}{4 d(d-1)} R\right)^{n},
\end{gathered}
$$

and $\kappa_{n}(d)$ determined through (27). Applying (13), we then find the transverse vector heat kernel coefficients for general endomorphisms as well. Table 2 shows our results for the first few vector heat kernels $(E=0)$ and for a selection of dimensions.

\subsection{Transverse traceless tensors}

Turning to the heat kernel coefficients for transverse traceless symmetric tensors, we first need to derive the Green's function. Just as in the case of transverse vectors, it is important to notice that the differential equation for the corresponding Green's function $G_{Q, \mu \nu \rho^{\prime} \sigma^{\prime}}^{T T}$ takes the form

$$
\left(-\nabla^{2}+Q\right) G_{Q, \mu \nu \rho^{\prime} \sigma^{\prime}}^{T T}=\frac{g_{\mu\left(\rho^{\prime} g_{\sigma^{\prime}}\right) v}}{\sqrt{g}} \delta(x-y)
$$$$
\text { +longitudinal and trace terms, }
$$

where longitudinal terms include terms which are longitudinal with respect to at least one index but not necessarily all indices. Following the methods introduced previously we write the transverse traceless symmetric Green's function as

$G_{Q, \mu \nu \rho^{\prime} \sigma^{\prime}}^{T T}=\mathcal{P}_{\mu \nu}^{\alpha \beta} \mathcal{P}_{\rho^{\prime} \sigma^{\prime}}^{\gamma^{\prime} \delta^{\prime}}\left(\mathcal{R}_{\alpha \gamma^{\prime}} \mathcal{R}_{\beta \delta^{\prime}} S^{T T}\right)$

The transverse traceless tensor projector is given by $[75,78]$ 


$$
\begin{aligned}
\mathcal{P}_{\mu \nu}{ }^{\alpha \beta}= & \frac{1}{2} \frac{d-3}{d-2}\left[-g_{(\mu}^{\alpha} g_{\nu)}^{\beta}\left(\nabla^{2}-\frac{R}{d-1}\right)\right. \\
& \times\left(\nabla^{2}-\frac{2 R}{d(d-1)}\right) \\
& +\frac{g_{\mu \nu} g^{\alpha \beta}}{d-1}\left(\nabla^{4}-\frac{R}{d} \nabla^{2}+\frac{2 R^{2}}{d^{2}(d-1)}\right) \\
& -\frac{g^{\alpha \beta}}{d-1} \nabla_{(\mu} \nabla_{\nu)}\left(\nabla^{2}+\frac{2 R}{d}\right) \\
& +2 \nabla_{(\mu}\left(\nabla^{2}+\frac{R}{d(d-1)}\right) g_{v)}^{(\alpha} \nabla^{\beta)} \\
& -\frac{d-2}{d-1} \nabla_{(\mu} \nabla_{\nu)} \nabla^{(\alpha} \nabla^{\beta)} \\
& \left.-\frac{g_{\mu \nu}}{d-1}\left(\nabla^{2}+\frac{2 R}{d}\right) \nabla^{(\alpha} \nabla^{\beta)}\right],
\end{aligned}
$$

and it satisfies

$$
\begin{aligned}
& \mathcal{P}_{\mu \nu}{ }^{\alpha \beta} g_{\alpha \beta} S(\sigma)=g^{\mu \nu} \mathcal{P}_{\mu \nu}{ }^{\alpha \beta} T_{\alpha \beta}=0, \\
& \quad\left[\nabla^{2}, \mathcal{P}_{\mu \nu}{ }^{\alpha \beta}\right] T_{\alpha \beta}=0, \\
& \mathcal{P}_{\mu \nu}{ }^{\alpha \beta} \nabla_{\alpha} T_{\beta}=\mathcal{P}_{\mu \nu}{ }^{\alpha \beta} \nabla_{\beta} T_{\alpha}=\nabla^{\mu} \mathcal{P}_{\mu \nu}{ }^{\alpha \beta} T_{\alpha \beta} \\
& \quad=\nabla^{\nu} \mathcal{P}_{\mu \nu}{ }^{\alpha \beta} T_{\alpha \beta}=0, \\
& \mathcal{P}_{\mu \nu}{ }^{\rho \sigma} \mathcal{P}_{\rho \sigma}{ }^{\alpha \beta} T_{\alpha \beta}=-\frac{1}{2} \frac{d-3}{d-2} \mathcal{P}_{\mu \nu}{ }^{\alpha \beta}\left(\nabla^{2}-\frac{2 R}{d(d-1)}\right) \\
& \quad \times\left(\nabla^{2}-\frac{R}{d-1}\right) T_{\alpha \beta}
\end{aligned}
$$

for an arbitrary tensor $T_{\alpha \beta}$. The Green's function can now be determined in a very similar fashion to the transverse vector Green's function. Firstly, we contract (53) with $\mathcal{P}_{\lambda \eta}{ }^{\mu \nu} \mathcal{P}_{\kappa^{\prime} \chi^{\prime}}{ }^{\rho^{\prime} \sigma^{\prime}}$. Making use of the relations for the transverse traceless tensor projector, the longitudinal and trace terms on the right-hand side vanish and we get

$$
\begin{aligned}
\mathcal{P}_{\lambda \eta}{ }^{\mu \nu} \mathcal{P}_{\kappa^{\prime} \chi^{\prime}}{ }^{\rho^{\prime} \sigma^{\prime}}\left(-\nabla^{2}+Q\right)\left(\nabla^{2}-\frac{2 R}{d(d-1)}\right)^{2} \\
\quad \times\left(\nabla^{2}-\frac{R}{d-1}\right)^{2}\left(\mathcal{R}_{\mu \rho^{\prime}} \mathcal{R}_{\nu \sigma^{\prime}} S^{T T}(\sigma)\right) \\
=4\left(\frac{d-2}{d-3}\right)^{2} \mathcal{P}_{\lambda \eta}{ }^{\mu \nu} \mathcal{P}_{\kappa^{\prime} \chi^{\prime}} \rho^{\prime} \sigma^{\prime} \\
\quad \times\left(\mathcal{R}_{\mu \rho^{\prime}} \mathcal{R}_{\nu \sigma^{\prime}} \frac{1}{\sqrt{g}} \delta(x-y)\right) .
\end{aligned}
$$

Using (37), this can be brought into the form

$$
\begin{aligned}
& \mathcal{P}_{\lambda \eta}{ }^{\mu \nu} \mathcal{P}_{\kappa^{\prime} \chi^{\prime}}{ }^{\rho^{\prime} \sigma^{\prime}}\left[\mathcal{R}_{\mu \rho^{\prime}} \mathcal{R}_{v \sigma^{\prime}}\left(-\nabla^{2}+Q-\frac{2 R}{d(d-1)}\right) \nabla^{4}\right. \\
& \left.\quad \times\left(\nabla^{2}-\frac{(d-2) R}{d(d-1)}\right)^{2} S^{T T}(\sigma)\right]
\end{aligned}
$$

$$
=4\left(\frac{d-2}{d-3}\right)^{2} \mathcal{P}_{\lambda \eta}{ }^{\mu \nu} \mathcal{P}_{\kappa^{\prime} \chi^{\prime}} \rho^{\prime} \sigma^{\prime}\left[\mathcal{R}_{\mu \rho^{\prime}} \mathcal{R}_{\nu \sigma^{\prime}} \frac{1}{\sqrt{g}} \delta(x-y)\right]
$$

from which we deduce that $S^{T T}(\sigma)$ obeys the differential equation

$$
\begin{aligned}
& \left(-\nabla^{2}+Q-\frac{2 R}{d(d-1)}\right) \nabla^{4}\left(\nabla^{2}-\frac{(d-2) R}{d(d-1)}\right)^{2} S^{T T}(\sigma) \\
& =\frac{4}{\sqrt{g}}\left(\frac{d-2}{d-3}\right)^{2} \delta(x-y) .
\end{aligned}
$$

The differential equation is solved by

$$
S^{T T}(\sigma)=4\left(\frac{d-2}{d-3}\right)^{2} G_{\chi_{1}, \chi_{2}, \chi_{2}, \chi_{3}, \chi_{3}}(\sigma)
$$

where we have introduced

$$
\begin{aligned}
G_{\chi_{1}, \chi_{2}, \chi_{2}, \chi_{3}, \chi_{3}}(\sigma) & =\frac{\partial}{\partial \chi_{2}} \frac{\partial}{\partial \chi_{3}} G_{\chi_{1}, \chi_{2}, \chi_{3}}(\sigma), \\
G_{\chi_{1}, \chi_{2}, \chi_{3}}(\sigma) & =\frac{G_{\chi_{1}, \chi_{3}}(\sigma)-G_{\chi_{1}, \chi_{2}}(\sigma)}{\chi_{2}-\chi_{3}},
\end{aligned}
$$

together with (21) and (40), and evaluated for

$$
\chi_{1}=Q-\frac{2 R}{d(d-1)}, \quad \chi_{2}=0, \quad \chi_{3}=\frac{d-2}{d(d-1)} R .
$$

To calculate the trace of $G_{Q, \mu \nu \rho^{\prime} \sigma^{\prime}}^{T T}$ in the coincidence limit, we first expand the term $\mathcal{R}_{\mu \rho^{\prime}} \mathcal{R}_{\nu \sigma^{\prime}} S^{T T}(\sigma)$ as before. Since we are now acting with eight covariant derivatives on this expression, see (60), we have to expand it up to order eight in $\sigma_{\mu}$. The relevant expression is lengthy and given in Appendix A, see (A4), where we also introduce

$$
S^{T T}(\sigma)=\sum_{n=0}^{\infty} S_{n}^{T T} \sigma^{n}
$$

Acting with the transverse tensor projectors and contracting the result with $g^{\mu\left(\rho^{\prime}\right.} g^{\left.\sigma^{\prime}\right) v}$, we obtain

$$
\begin{aligned}
g^{\mu\left(\rho^{\prime}\right.} g^{\left.\sigma^{\prime}\right) \nu} G_{Q, \mu \nu \rho^{\prime} \sigma^{\prime}}^{T T}(0) & \\
= & \frac{(d-3)^{2}(d+1)(d+2)}{d-2}\left[\frac{(13 d-32) R^{3}}{140(d-1)^{2} d^{2}} S_{1}^{T T}\right. \\
& +\frac{(d-6)(7 d-8) R^{2}}{20(d-1)^{2} d} S_{2}^{T T} \\
& \left.+\frac{3(3-2 d)(d+4) R}{2(d-1)} S_{3}^{T T}+3 d(d+4)(d+6) S_{4}^{T T}\right] .
\end{aligned}
$$

Inserting the expansion coefficients for $S^{T T}(\sigma)$ and using (26), we finally arrive at

$$
g^{\mu\left(\rho^{\prime}\right.} g^{\left.\sigma^{\prime}\right) \nu} G_{Q, \mu \nu \rho^{\prime} \sigma^{\prime}}^{T T}(0)
$$




$$
\begin{aligned}
= & \frac{2^{-d-1}(d-1) \pi^{-\frac{d}{2}}}{\Gamma\left(\frac{d}{2}\right)((d-1) Q-R)}\left(\frac{R}{d(d-1)}\right)^{d / 2} \\
& \times\left[\frac{\Gamma(d+2)\left((d-1) d(d+2) Q-\left(d^{2}+4\right) R\right)}{d((d-1) d Q-2 R)}\right. \\
& +\frac{\pi(d-2)(d+1)((d-1) d Q+(d-2) R)}{\sin \left(\frac{\pi d}{2}\right) R} \\
& \left.\sum_{k=0}^{\infty} \kappa_{k}(d)\left(-\widetilde{\xi}^{2}\right)^{d / 2-k-1}\right] .
\end{aligned}
$$

where

$$
\widetilde{\xi}=\frac{1}{2} \sqrt{\left(-\frac{4(d-1) Q}{R}+d-2\right) d+9}
$$

Just as before, we now choose the endomorphism in order to simplify the sum. Taking

$$
\begin{aligned}
\bar{E} & =-\frac{9-2 d+d^{2}}{4 d(d-1)} R, \\
-\widetilde{\xi}^{2} & =d(d-1) \frac{m^{2}}{R},
\end{aligned}
$$

and using (67) and the geometric series to expand the denominators in the large $m$ limit, we find

$$
\begin{aligned}
& \left.g^{\mu\left(\rho^{\prime}\right.} g^{\left.\sigma^{\prime}\right) v} G_{Q, \mu \nu \rho^{\prime} \sigma^{\prime}}^{T T}(0)\right|_{E=\bar{E}} \\
& =\frac{2^{-d-2} \pi^{-d / 2}}{m^{2} R \Gamma\left(\frac{d}{2}-1\right)}\left(\frac{R}{d(d-1)}\right)^{1+d / 2} \\
& \times \sum_{n=0}^{\infty}\left(-\frac{(d-3)^{2}}{4 d(d-1)} \frac{R}{m^{2}}\right)^{n} \\
& \times\left[\frac{\pi d\left(d^{2}-1\right)\left(4(d-1) d m^{2}+(d+1)^{2} R\right)}{\sin \left(\frac{\pi d}{2}\right) R}\right. \\
& \times \sum_{k=0}^{\infty} \kappa_{k}(d)\left(\frac{d(d-1) m^{2}}{R}\right)^{d / 2-k-1} \\
& +\frac{\Gamma(d+2)\left(4(d-1) d(d+2) m^{2}+(d((d-4) d+5)+2) R\right)}{(d-2) d m^{2}} \\
& \left.\times \sum_{m=0}^{\infty}\left(-\frac{d-1}{4 d} \frac{R}{m^{2}}\right)^{m}\right] \\
& =\frac{1}{(4 \pi)^{d / 2}}\left(\frac{R}{d(d-1)}\right)^{d / 2} \frac{1}{\Gamma(d / 2-1)} \sum_{k=0}^{\infty}\left(\frac{R}{m^{2}}\right)^{k} \\
& \times\left[\frac{\Gamma(d+2)}{(d-2)}\left(\frac{d+2}{d m^{2}}+\frac{(2+d(5+d(d-4))) R}{4 d^{2}(d-1) m^{4}}\right)\right. \\
& \times \sum_{\ell=0}^{k}\left(-\frac{(d-3)^{2}}{4 d(d-1)}\right)^{\ell}\left(-\frac{d-1}{4 d}\right)^{k-\ell} \\
& +\frac{\pi(d(d-1))^{-k}}{\sin (d \pi / 2)}\left(\frac{(d+1)^{3}}{4 m^{2}}+\frac{d\left(d^{2}-1\right)}{R}\right) \\
& \left.\times\left(\frac{R}{d(d-1) m^{2}}\right)^{1-d / 2} \sum_{\ell=0}^{k} \kappa_{\ell}(d)\left(-\frac{(d-3)^{2}}{4}\right)^{k-\ell}\right] .
\end{aligned}
$$

Note that in the last step sums have been reorganised in order to combine powers of $m$. As before, using (68) and the specific endomorphism (67), the heat kernel coefficients are found to be

$$
\begin{aligned}
b_{2 n}^{(2)}(\bar{E}) & =\frac{1}{\Gamma(1+n-d / 2)}\left(\frac{R}{d(d-1)}\right)^{n} \\
\times & \frac{\pi(d+1)}{\Gamma(d / 2-1) \sin (d \pi / 2)} \\
\times & {\left[\sum_{\ell=0}^{n} \kappa_{\ell}(d)\left(-\frac{(d-3)^{2}}{4}\right)^{n-\ell}+\frac{(d+1)^{2}}{4}\right.} \\
\times & \left.\sum_{\ell=0}^{n-1} \kappa_{\ell}(d)\left(-\frac{(d-3)^{2}}{4}\right)^{n-1-\ell}\right], \\
c_{d+2 n}^{(2)} & (\bar{E})=\frac{2}{\Gamma(1+n)}\left(\frac{R}{d(d-1)}\right)^{d / 2} \frac{\Gamma(d+2)}{\Gamma(d / 2)} R^{n} \\
\times & {\left[\frac{d+2}{4 d} \sum_{\ell=0}^{n}\left(-\frac{(d-3)^{2}}{4 d(d-1)}\right)^{\ell}\left(-\frac{d-1}{4 d}\right)^{n-\ell}\right.} \\
+ & \frac{2+d(5+d(d-4))}{16 d^{2}(d-1)} \\
\times & \left.\times \sum_{\ell=0}^{n-1}\left(-\frac{(d-3)^{2}}{4 d(d-1)}\right)^{\ell}\left(-\frac{d-1}{4 d}\right)^{n-1-\ell}\right],
\end{aligned}
$$

with $\kappa_{n}(d)$ determined through (27). As has been observed for the scalar and transverse vector heat kernel coefficients, the coefficients $b_{2 n}^{(2)}$ are identified from (68) as the terms linear in $\kappa_{i}(d)$ while the coefficients $c_{d+2 n}^{(2)}$ are found by setting all $\kappa_{i}(d)$ to zero. The heat kernel coefficients for arbitrary endomorphisms can be found using (13). Table 3 shows our results for the first few tensor heat kernels $(E=0)$ and for a selection of dimensions.

As a final remark, we emphasize that the explicit expressions for the heat kernel coefficients of scalar (30), transverse vectors (52), and transverse traceless tensors (69), are easy to evaluate and implement for arbitrary endomorphism and arbitrary dimension, including non-integer ones. For example, the first 100 heat kernel coefficients (69) at vanishing endomorphism in $d=4$ are found within $20 \mathrm{~s}$ on a $3 \mathrm{GHz}$ thread, also using (13) and the coefficients (27).

\subsection{Unconstrained fields}

In the literature, heat kernel coefficients are often calculated for unconstrained fields. A relation between the heat kernels of constrained fields and unconstrained fields can be derived by decomposing unconstrained fields into constrained fields.

For vectors, the unconstrained vector field $v_{\mu}$ can be decomposed into a transverse vector field $v_{\mu}^{T}$ and a longitudinal part $\nabla_{\mu} \eta$ through 
Table 3 The tensor heat kernel coefficients for different integer dimensions and vanishing endomorphism

\begin{tabular}{llllll}
\hline & $d=2$ & $d=3$ & $d=4$ & $d=5$ & $d=6$ \\
\hline$b_{0}^{(2)}$ & 0 & 2 & 5 & 9 & 14 \\
$c_{d}^{(2)}$ & $3 R$ & $\frac{20}{3 \sqrt{6 \pi}} R^{3 / 2}$ & $\frac{5}{8} R^{2}$ & $\frac{21}{25 \sqrt{5 \pi}} R^{5 / 2}$ & $\frac{14}{225} R^{3}$ \\
$b_{2}^{(2)}$ & $-3 R$ & $-\frac{5}{3} R$ & $-\frac{5}{6} R$ & 0 & $\frac{14}{15} R$ \\
$c_{d+2}^{(2)}$ & $3 R^{2}$ & $\frac{8}{3 \sqrt{6 \pi}} R^{5 / 2}$ & $\frac{5}{36} R^{3}$ & $\frac{3}{25 \sqrt{5 \pi}} R^{7 / 2}$ & $\frac{7}{1125} R^{4}$ \\
$b_{4}^{(2)}$ & $-3 R^{2}$ & $-\frac{13}{12} R^{2}$ & $-\frac{271}{432} R^{2}$ & $-\frac{81}{200} R^{2}$ & $-\frac{21}{225} R^{2 / 2}$ \\
$c_{d+4}^{(2)}$ & $\frac{3}{2} R^{3}$ & $\frac{5}{9 \sqrt{6 \pi}} R^{7 / 2}$ & $\frac{5}{288} R^{4}$ & $-\frac{81}{1000} R^{3}$ & $\frac{29}{16875} R^{5}$ \\
$b_{6}^{(2)}$ & $-\frac{3}{2} R^{3}$ & $-\frac{7}{24} R^{3}$ & $-\frac{7249}{54432} R^{3}$ & $\frac{29}{40000 \sqrt{5 \pi}} R^{11 / 2}$ & $-\frac{3347}{60750} R^{3}$ \\
$c_{d+6}^{(2)}$ & $\frac{1}{2} R^{4}$ & $\frac{13}{162 \sqrt{6 \pi}} R^{9 / 2}$ & $\frac{25}{15552} R^{5}$ & $-\frac{729}{80000} R^{4}$ & $\frac{13}{303750} R^{6}$ \\
$b_{8}^{(2)}$ & $-\frac{1}{2} R^{4}$ & $-\frac{29}{576} R^{4}$ & $-\frac{22571}{1306368} R^{4}$ & $\frac{133 / 2}{3200000 \sqrt{5 \pi}} R^{13 / 2}$ \\
$c_{d+8}^{(2)}$ & $\frac{1}{8} R^{5}$ & $\frac{35}{3888 \sqrt{6 \pi}} R^{11 / 2}$ & $\frac{5}{41472} R^{6}$ & $-\frac{729}{1000000} R^{5}$ \\
$b_{10}^{(2)}$ & $-\frac{1}{8} R^{5}$ & $-\frac{37}{5760} R^{5}$ & $-\frac{20117}{12317184} R^{5}$ & $\frac{106429}{3825000} R^{4}$ \\
$c_{d+10}^{(2)}$ & $\frac{1}{40} R^{6}$ & $\frac{97}{116640 \sqrt{6 \pi}} R^{13 / 2}$ & $\frac{17}{2239488} R^{7}$ & $\frac{49}{45562500} R^{7}$ \\
\hline
\end{tabular}

$v_{\mu}=v_{\mu}^{T}+\nabla_{\mu} \eta$

Note that the first mode of the scalar field $\eta$ is constant and does not contribute to $v_{\mu}$. Hence, this mode must be excluded later. Considering the Laplacian $-\nabla^{2}$ acting on $v_{\mu}$, we can write

$$
\begin{aligned}
-\nabla^{2} v_{\mu} & =-\nabla^{2}\left(v_{\mu}^{T}+\nabla_{\mu} \eta\right) \\
& =-\nabla^{2} v_{\mu}^{T}+\nabla_{\mu}\left(-\nabla^{2}-\frac{R}{d}\right) \eta
\end{aligned}
$$

on the sphere. It follows that the eigenvalue spectrum of $-\nabla^{2}$ acting on an unconstrained vector field is the sum of two parts. The first part is the eigenvalue spectrum of the Laplacian acting on a transverse vector field. The second part is the eigenvalue spectrum of the Laplacian acting on a scalar field shifted by $-R / d$ and the first mode being excluded. Thus,

$\operatorname{Tr}_{V} e^{t \nabla^{2}}=\operatorname{Tr}_{1} e^{t \nabla^{2}}+\operatorname{Tr}_{0}^{\prime} e^{t\left(\nabla^{2}+R / d\right)}$,

where primes at the trace denote the exclusion of lowest modes and $\operatorname{Tr}_{V}$ denotes the trace of an unconstrained vector field. This allows the computation of heat kernel coefficients for unconstrained fields using our results. We get

$$
\begin{aligned}
\operatorname{Tr}_{V} e^{t \nabla^{2}} & =\frac{\text { Vol }}{(4 \pi t)^{d / 2}}\left[d+\frac{d}{6} R t+\frac{5 d^{3}-7 d^{2}+6 d-60}{360(d-1) d} R^{2} t^{2}\right. \\
& +\frac{35 d^{5}-112 d^{4}+187 d^{3}-1370 d^{2}+852 d-1008}{45360(d-1)^{2} d^{2}} R^{3} t^{3} \\
& +\frac{R^{4} t^{4}}{5443200(d-1)^{3} d^{3}}\left(175 d^{7}-945 d^{6}+2389 d^{5}\right. \\
& -15711 d^{4}+23464 d^{3} \\
& \left.-35436 d^{2}+59760 d-62640\right)
\end{aligned}
$$

$$
\begin{aligned}
& +\frac{R^{5} t^{5}}{359251200(d-1)^{4} d^{4}}\left(385 d^{9}-3080 d^{8}\right. \\
& +10714 d^{7}-68156 d^{6}+168793 d^{5} \\
& -308084 d^{4}+858996 d^{3}-958944 d^{2}+857232 d \\
& \left.-798336)+\mathcal{O}(R t)^{6}\right] .
\end{aligned}
$$

As anticipated, we observe from the result that all coefficients $\tilde{c}_{d+2 n}^{(1)}$ and $c_{d+2 n}^{(1)}$ vanish for unconstrained fields, see (5) and (9).

Turning to tensors and using the York decomposition, we decompose an unconstrained symmetric tensor field $T_{\mu \nu}$ into

$$
\begin{aligned}
T_{\mu \nu}= & T_{\mu \nu}^{T T}+\nabla_{\mu} \xi_{\nu}+\nabla_{\nu} \xi_{\mu} \\
& +\left(\nabla_{\mu} \nabla_{\nu}-\frac{1}{d} g_{\mu \nu} \nabla^{2}\right) \sigma+\frac{1}{d} g_{\mu \nu} \eta
\end{aligned}
$$

with $T_{\mu \nu}^{T T}$ being a transverse traceless symmetric tensor, $\xi_{\mu}$ being a transverse vector, and $\sigma$ and $\eta$ being scalars. As in the case of vector fields, not all modes of this decomposition contribute to $T_{\mu \nu}^{T T}$. These modes which have to be excluded are (i) the $d(d+1) / 2$ Killing vectors of $\xi_{\mu}$ which satisfy $\nabla_{\mu} \xi_{\nu}+\nabla_{\nu} \xi_{\mu}=0$ and originate from the lowest transverse vector modes, (ii) the constant (lowest) mode of $\sigma$, and (iii) the $d+1$ scalars of the second lowest modes of $\sigma$ (see Table 4 for the multiplicities of these modes) corresponding to the proper conformal Killing vectors $\zeta_{\mu}=\nabla_{\mu} \sigma$, which satisfy $\nabla_{\mu} \zeta_{\nu}+\nabla_{\nu} \zeta_{\mu}-\frac{2}{d} g_{\mu \nu} \nabla^{\tau} \zeta_{\tau}=0$ [79] (see also [80]). Acting with the Laplacian on $T_{\mu \nu}$, we use 


$$
\begin{aligned}
& \nabla^{2}\left(\nabla_{\mu} \xi_{\nu}+\nabla_{\nu} \xi_{\mu}\right) \\
& =2 \nabla_{(\mu}\left(-\nabla^{2}-\frac{d+1}{d(d-1)} R\right) \xi_{v)} \\
& -\nabla^{2}\left(\nabla_{\mu} \nabla_{\nu}-\frac{1}{d} g_{\mu \nu} \nabla^{2}\right) \sigma \\
& =\left(\nabla_{\mu} \nabla_{\nu}-\frac{1}{d} g_{\mu \nu} \nabla^{2}\right)\left(-\nabla^{2}-\frac{2}{d-1} R\right) \sigma
\end{aligned}
$$

to arrive at

$$
\begin{aligned}
- & \nabla^{2} T_{\mu \nu} \\
= & -\nabla^{2} T_{\mu \nu}^{T T}+2 \nabla_{(\mu}\left(-\nabla^{2}-\frac{d+1}{d(d-1)} R\right) \xi_{\nu)} \\
& +\left(\nabla_{\mu} \nabla_{\nu}-\frac{1}{d} g_{\mu \nu} \nabla^{2}\right)\left(-\nabla^{2}-\frac{2}{d-1} R\right) \sigma \\
& -\frac{1}{d} g_{\mu \nu} \nabla^{2} \eta .
\end{aligned}
$$

This implies

$$
\begin{aligned}
\operatorname{Tr}_{T} e^{t \nabla^{2}}= & \operatorname{Tr}_{2} e^{t \nabla^{2}}+\operatorname{Tr}_{1}^{\prime} e^{t\left(\nabla^{2}+\frac{d+1}{d(d-1)} R\right)}+\operatorname{Tr}_{0}^{\prime \prime} e^{t\left(\nabla^{2}+\frac{2}{d-1} R\right)} \\
& +\operatorname{Tr}_{0} e^{t \nabla^{2}}
\end{aligned}
$$

where $\operatorname{Tr}_{T}$ denotes the trace of the Laplacian w.r.t. an unconstrained symmetric tensor field and two primes denote the exclusion of the two lowest modes. Using this and our results for the heat kernel coefficients of constrained fields we find

$$
\begin{aligned}
\operatorname{Tr}_{T} e^{t \nabla^{2}}=\frac{\text { Vol }}{(4 \pi t)^{d / 2}}\left[\frac{d(d+1)}{2}+\frac{d(d+1)}{12} R t\right. \\
+\frac{5 d^{4}-2 d^{3}-d^{2}-114 d-240}{720(d-1) d} R^{2} t^{2} \\
+\frac{35 d^{6}-77 d^{5}+75 d^{4}-2443 d^{3}-3542 d^{2}+1104 d-4032}{90720(d-1)^{2} d^{2}} R^{3} t^{3} \\
+\frac{R^{4} t^{4}}{10886400(d-1)^{3} d^{3}}\left(175 d^{8}-770 d^{7}+1444 d^{6}\right. \\
+25922 d^{5}-9887 d^{4} \\
\left.+13588 d^{3}+188844 d^{2}+742320 d+172800\right) \\
+\frac{R^{5} t^{5}}{718502400(d-1)^{4} d^{4}}\left(385 d^{10}-2695 d^{9}+7634 d^{8}\right. \\
-103642 d^{7}+100637 d^{6}-8875 d^{5} \\
+2850880 d^{4}+8146092 d^{3}+7406448 d^{2} \\
\left.+18339840 d+8211456)+\mathcal{O}(R t)^{6}\right] .
\end{aligned}
$$

Note that all coefficients $\tilde{c}_{d+2 n}^{(2)}$ and $c_{d+2 n}^{(2)}$ vanish for unconstrained fields. This completes the derivation of heat kernel coefficients.
Table 4 The eigenvalues $\lambda_{\ell}^{s}$ and their multiplicities $D_{\ell}^{s}$ for spin 0 (scalar), spin 1 (transverse vector), and spin 2 (transverse traceless tensor) fields of the operator $-\nabla^{2}$ on the sphere (taken from $[81,82]$ )

\begin{tabular}{lll}
\hline Spin $s$ & Eigenvalue $\lambda_{\ell}^{s}$ & Multiplicity $D_{\ell}^{s}$ \\
\hline 0 & $\frac{\ell(\ell+d-1)}{d(d-1)} R ; \ell=0,1,2, \ldots$ & $\frac{(2 \ell+d-1)(\ell+d-2) !}{\ell !(d-1) !}$ \\
1 & $\frac{\ell(\ell+d-1)-1}{d(d-1)} R ; \ell=1,2,3, \ldots$ & $\frac{\ell(\ell+d-1)(2 \ell+d-1)(\ell+d-3) !}{(d-2) !(\ell+1) !}$ \\
2 & $\frac{\ell(\ell+d-1)-2}{d(d-1)} R ; \ell=2,3,4, \ldots$ & $\frac{(d+1)(d-2)(\ell+d)(\ell-1)(2 \ell+d-1)(\ell+d-3) !}{2(d-1) !(\ell+1) !}$ \\
\hline
\end{tabular}

\section{Heat kernels from spectral sums}

In this section, we compute heat kernel coefficients with the help of spectral sums and the Euler-Maclaurin formula. This serves as an independent consistency check for findings in the previous section. We also find new expressions for certain heat kernel coefficients in the form of spectral integrals.

\subsection{Spectral sum technique}

The eigenspectrum of the Laplacian $-\nabla^{2}$ is known on the sphere $[81,82]$ and can be used to calculate the heat kernel coefficients, e.g. $[49,71,83]$. Specifically, for different spins $s$, the eigenfunctions $\phi_{\ell}^{s}$ satisfy the eigenvalue equation

$-\nabla^{2} \phi_{\ell}^{s}(x)=\lambda_{\ell}^{s} \phi_{\ell}^{s}(x)$

where $\lambda_{\ell}^{s}$ are the eigenvalues and all vector and tensor indices have been suppressed. In Table 4 we show the eigenvalues and their multiplicities $D_{\ell}^{s}$ for spin 0 , spin 1 , and spin 2 fields on the sphere. The eigenfunctions can be chosen to be orthonormal,

$\int \mathrm{d}^{d} x \sqrt{g} \phi_{\ell, n}^{s *}(x) \phi_{k, m}^{s}(x)=\delta_{\ell, k} \delta_{n, m}$,

where we use the indices $n$ and $m$ to distinguish eigenfunctions with equal eigenvalues. Further, they satisfy the completeness relation

$\sum_{\ell, n} \phi_{\ell, n}^{s *}(x) \phi_{\ell, n}^{s}(y)=\frac{\delta(x-y)}{\sqrt{g}}$.

With this at hand, and for vanishing endomorphisms, we may express the trace of the heat kernel as a multiplicity-weighted sum of its eigenvalues,

$$
\begin{aligned}
\operatorname{Tr}_{s} U_{0}(t, \sigma) & =\sum_{\ell, n} \int \mathrm{d}^{d} x \sqrt{g} \phi_{\ell, n}^{s *}(x) e^{t \nabla^{2}} \phi_{\ell, n}^{s}(x) \\
& =\sum_{\ell} D_{\ell}^{s} e^{-t \lambda_{\ell}^{s}}
\end{aligned}
$$


In principle, the spectral sum representation allows the extraction of heat kernel coefficients by projection

$$
\begin{aligned}
b_{2 n}^{(s)} & =\left.\frac{(4 \pi)^{d / 2}}{\operatorname{Vol}} \frac{1}{n !} \frac{\mathrm{d}^{n}}{\mathrm{~d} t^{n}}\left(t^{d / 2} \sum_{\ell} D_{\ell}^{s} e^{-t \lambda_{\ell}^{s}}\right)\right|_{t=0}, \\
c_{d+2 n}^{(s)} & =\left.\frac{(4 \pi)^{d / 2}}{\operatorname{Vol}} \frac{1}{n !} \frac{\mathrm{d}^{n}}{\mathrm{~d} t^{n}}\left(\sum_{\ell} D_{\ell}^{s} e^{-t \lambda_{\ell}^{s}}\right)\right|_{t=0} .
\end{aligned}
$$

Note that in even dimension $d=2 m$, the coefficients $b_{2(m+n)}$ and $c_{d+2 n}$ cannot be distinguished. In practice, to find explicit expressions, the spectral sum in (83) and (84) is now approximated systematically using the Euler-Maclaurin formula

$$
\begin{aligned}
\sum_{\ell=a}^{b} f(\ell)= & \int_{a}^{b} \mathrm{~d} \ell f(\ell)+\frac{1}{2}(f(a)+f(b)) \\
& +\sum_{k=1}^{n} \frac{B_{2 k}}{(2 k) !}\left(f^{(2 k-1)}(b)-f^{(2 k-1)}(a)\right) \\
& +R_{2 n+1},
\end{aligned}
$$

where $B_{n}$ are the Bernoulli numbers (recall that $B_{n}=0$ for odd $n$ except $n=1)$ and $f^{(n)}(\ell) \equiv \frac{\mathrm{d}^{n}}{\mathrm{~d} \ell^{n}} f(\ell)$; see $e . g$. [49] for a derivation of (85). An approximation of the EulerMaclaurin formula at order $2 n$ corresponds to dropping the remainder part

$R_{2 n+1}=\frac{1}{(2 n+1) !} \int_{a}^{b} \mathrm{~d} \ell f^{(2 n+1)}(\ell) P_{2 n+1}(\ell)$,

where $P_{n}(\ell)=B_{n}(\ell-\lfloor\ell\rfloor)$ are periodized versions of the Bernoulli polynomials $B_{n}(\ell)$. An upper bound for the remainder is given by

$\left|R_{2 n+1}\right| \leq \frac{2}{(2 \pi)^{2 n}} \int_{a}^{b} \mathrm{~d} \ell\left|f^{(2 n+1)}(\ell)\right|$.

In the case at hand, we have $a=s, 1 / b=0$, and $f(\ell)=$ $D_{\ell}^{s} e^{-t \lambda_{\ell}^{s}}$, with eigenvalues and multiplicities as in Table 4 . The eigenvalues are quadratic polynomials in $\ell$ and imply that the leading small $t$ behaviour of the remainder is controlled by the leading large $\ell$ behaviour of the integrand, hence $\ell^{2} \sim t^{-1}$. In this limit, multiplicities of eigenmodes for scalars, transverse vectors, and transverse traceless tensors scale according to $D_{\ell}^{s} \sim \ell^{d-1} \sim t^{(1-d) / 2}$. Together with the elementary integral $\int_{a}^{\infty} \mathrm{d} \ell \exp -t \ell^{2}=\sqrt{\frac{\pi}{4 t}}-a+\mathcal{O}\left(t a^{3}\right)$ we find a lower bound for the leading power in $t$ contained in the remainder,
$R_{2 n+1}=\mathcal{O}\left(t^{n+(1-d) / 2}\right)$.

Therefore, we may expect that truncating the Euler-Maclaurin formula (85) to a finite order $2 n$ and leaving out the remainder $R_{2 n+1}$ reproduces the series expansion in the proper time parameter for the heat kernel (83) up to all orders including $t^{(2 n-d) / 2}$. This corresponds to coefficients up to order $n$ in Ricci curvature $\sim \frac{\mathrm{Vol}}{(4 \pi t)^{d / 2}}(R t)^{n}$ in the heat kernel expansion (11) with coefficients (84). Below, we also show that the bound is exhaustive. Finally, we note that the boundary and derivative terms $f(a), f(b)$, and $\left.f^{(2 k-1)}(\ell)\right|_{a} ^{b}$ only generate contributions proportional to integer positive powers in $t$, in any dimension.

\subsection{Results from Euler-Maclaurin}

The Euler-Maclaurin formula (85) is most efficient in settings where the spectral integrals $\int_{a}^{b} \mathrm{~d} \ell f(\ell)$ can be solved in closed form. For our setting, this is the case in integer dimensions where the multiplicities are simple polynomials. The bound (88) for the remainder ensures that approximations improve with increasing order. Here, we exploit these features to cross-check results for heat kernel coefficients, order-by-order.

Putting our rationale to work for the scalar heat kernel coefficients in integer dimensions by using the expression (83) together with (85), we find

$$
\begin{aligned}
& \left.\operatorname{Tr}_{0} U_{0}(t, \sigma)\right|_{d=2} \\
& =\frac{\operatorname{Vol}}{4 \pi t}\left(1+\frac{R t}{6}+\frac{R^{2} t^{2}}{60}+\frac{R^{3} t^{3}}{630}+\frac{R^{4} t^{4}}{5040}+\frac{R^{5} t^{5}}{27720}\right. \\
& \left.\quad+\mathcal{O}(R t)^{6}\right) \\
& \left.\operatorname{Tr}_{0} U_{0}(t, \sigma)\right|_{d=3} \\
& =\frac{\operatorname{Vol}^{3 / 2}}{(4 \pi t)^{3 / 2}}\left(1+\frac{R t}{6}+\frac{R^{2} t^{2}}{72}+\frac{R^{3} t^{3}}{1296}+\frac{R^{4} t^{4}}{31104}\right. \\
& \left.\quad+\frac{R^{5} t^{5}}{933120}+\mathcal{O}(R t)^{6}\right), \\
& \left.\operatorname{Tr}_{0} U_{0}(t, \sigma)\right|_{d=4} \\
& =\frac{\operatorname{Vol}_{d}}{(4 \pi t)^{2}}\left(1+\frac{R t}{6}+\frac{29 R^{2} t^{2}}{2160}+\frac{37 R^{3} t^{3}}{54432}+\frac{149 R^{4} t^{4}}{6531840}\right. \\
& \left.\quad+\frac{179 R^{5} t^{5}}{431101440}+\mathcal{O}(R t)^{6}\right), \\
& \left.\operatorname{Tr}_{0} U_{0}(t, \sigma)\right|_{d=5}
\end{aligned}
$$




$$
\begin{aligned}
= & \frac{\mathrm{Vol}}{(4 \pi t)^{5 / 2}}\left(1+\frac{R t}{6}+\frac{R^{2} t^{2}}{75}+\frac{R^{3} t^{3}}{1500}+\frac{R^{4} t^{4}}{45000}\right. \\
& \left.+\frac{R^{5} t^{5}}{2250000}+\mathcal{O}(R t)^{6}\right) \\
\left.\operatorname{Tr}_{0} U_{0}(t, \sigma)\right|_{d=6} & \\
= & \frac{\mathrm{Vol}}{(4 \pi t)^{3}}\left(1+\frac{R t}{6}+\frac{R^{2} t^{2}}{75}+\frac{1139 R^{3} t^{3}}{1701000}+\frac{833 R^{4} t^{4}}{36450000}\right. \\
& \left.+\frac{137 R^{5} t^{5}}{267300000}+\mathcal{O}(R t)^{6}\right) .
\end{aligned}
$$

To obtain results for heat kernel coefficients up to order $n=5$ in the Ricci curvature, we confirm that an expansion of (85) up to order $2 n=10$ is required, in agreement with the estimate for the remainder term stated in (88). Also, results are in exact agreement with findings from the Green's function technique (see Table 1) and confirm that the leading contribution starts out as $t^{-d / 2}$.

The same analysis can now be done for transverse vectors and transverse traceless tensors. However, for these cases it is important to realise that in even dimensions we cannot distinguish between the contributions coming from the $b_{d+2 n}^{(s)}$ and the $c_{d+2 n}^{(s)}(n \geq 0)$ when we take the sum of the eigenvalues, see (84). Thus, in even dimension we can only compare their sum with the findings from spectral sums. Again, to find all coefficients $b_{2 m}^{(s)}$ and the $c_{2 m}^{(s)}$ up to $m=n$ we have to expand (85) up to including the order $2 n$, see (88). For the transverse vectors in even dimension and for expansion order $2 n=10$ we find using the spectral sum

$$
\begin{gathered}
\left.\operatorname{Tr}_{1} U_{0}(t, \sigma)\right|_{d=2} \\
=\frac{\mathrm{Vol}}{4 \pi t}\left(1+\frac{R t}{6}-\frac{R^{2} t^{2}}{40}-\frac{11 R^{3} t^{3}}{1008}\right. \\
\left.\quad-\frac{17 R^{4} t^{4}}{13440}+\frac{13 R^{5} t^{5}}{177408}+\mathcal{O}(R t)^{6}\right) \\
\left.\operatorname{Tr}_{1} U_{0}(t, \sigma)\right|_{d=4} \\
=\frac{\operatorname{Vol}}{(4 \pi t)^{2}}\left(3+\frac{R t}{4}-\frac{7 R^{2} t^{2}}{1440}-\frac{541 R^{3} t^{3}}{362880}\right. \\
\left.-\frac{157 R^{4} t^{4}}{2488320}+\frac{4019 R^{5} t^{5}}{2299207680}+\mathcal{O}(R t)^{6}\right) \\
\left.\operatorname{Tr}_{1} U_{0}(t, \sigma)\right|_{d=6} \\
=\frac{\operatorname{Vol}^{3}}{(4 \pi t)^{3}}\left(5+\frac{2 R t}{3}+\frac{7 R^{2} t^{2}}{360}-\frac{649 R^{3} t^{3}}{850500}\right.
\end{gathered}
$$

$$
\left.-\frac{24907 R^{4} t^{4}}{408240000}-\frac{5849 R^{5} t^{5}}{6735960000}+\mathcal{O}(R t)^{6}\right)
$$

Taking the sum $b_{d+2 n}^{(1)}+c_{d+2 n}^{(1)}$ from Table 2, the consistency of our results with the Geen's function method is confirmed. In odd dimensions, we can distinguish between the contributions of $b_{d+2 n}^{(1)}$ and $c_{d+2 n}^{(1)}$ from within the spectral sum technique, and the results up to order five in Ricci curvature

$$
\begin{aligned}
& \left.\operatorname{Tr}_{1} U_{0}(t, \sigma)\right|_{d=3} \\
& =\frac{\mathrm{Vol}}{(4 \pi t)^{3 / 2}}\left(2+\frac{2 R^{3 / 2} t^{3 / 2}}{3 \sqrt{6 \pi}}-\frac{R^{2} t^{2}}{9}+\frac{2 R^{5 / 2} t^{5 / 2}}{9 \sqrt{6 \pi}}\right. \\
& -\frac{2}{81} R^{3} t^{3}+\frac{R^{7 / 2} t^{7 / 2}}{27 \sqrt{6 \pi}} \\
& \left.-\frac{1}{324} R^{4} t^{4}+\frac{R^{9 / 2} t^{9 / 2}}{243 \sqrt{6 \pi}}-\frac{R^{5} t^{5}}{3645}+\mathcal{O}(R t)^{11 / 2}\right), \\
& \left.\operatorname{Tr}_{1} U_{0}(t, \sigma)\right|_{d=5} \\
& =\frac{\mathrm{Vol}}{(4 \pi t)^{5 / 2}}\left(4+\frac{7 R t}{15}-\frac{R^{2} t^{2}}{120}+\frac{R^{5 / 2} t^{5 / 2}}{25 \sqrt{5 \pi}}-\frac{R^{3} t^{3}}{160}\right. \\
& +\frac{R^{7 / 2} t^{7 / 2}}{125 \sqrt{5 \pi}}-\frac{17 R^{4} t^{4}}{23040} \\
& \left.+\frac{R^{9 / 2} t^{9 / 2}}{1250 \sqrt{5 \pi}}-\frac{R^{5} t^{5}}{18432}+\mathcal{O}(R t)^{11 / 2}\right) .
\end{aligned}
$$

fully agree with the corresponding results from the Green's function technique (see Table 2). Finally, for the transverse traceless tensor heat kernels in even dimensions we get from the spectral sum approximation

$$
\begin{aligned}
\operatorname{Tr}_{2} U_{0}(t, \sigma) & =0 \\
\operatorname{Tr}_{2} U_{0}(t, \sigma) & \left.\right|_{d=4} \\
= & \frac{\mathrm{Vol}}{(4 \pi t)^{2}}\left(5-\frac{5 R t}{6}-\frac{R^{2} t^{2}}{432}+\frac{311 R^{3} t^{3}}{54432}\right. \\
& \left.+\frac{109 R^{4} t^{4}}{1306368}-\frac{317 R^{5} t^{5}}{12317184}+\mathcal{O}(R t)^{6}\right), \\
\left.\operatorname{Tr}_{2} U_{0}(t, \sigma)\right|_{d=6} & \frac{\operatorname{Vol}^{3}}{(4 \pi t)^{3}}\left(14+\frac{14 R t}{15}-\frac{56 R^{2} t^{2}}{225}+\frac{433 R^{3} t^{3}}{60750}\right. \\
& \left.+\frac{6971 R^{4} t^{4}}{18225000}-\frac{28357 R^{5} t^{5}}{3007125000}+\mathcal{O}(R t)^{6}\right) .
\end{aligned}
$$


Similar to the case of transverse vectors, the consistency of these results with Table 3 is confirmed by taking the sum $b_{d+2 n}^{(2)}+c_{d+2 n}^{(2)}$. In odd dimensions we find

$$
\begin{aligned}
\operatorname{Tr}_{2} & \left.U_{0}(t, \sigma)\right|_{d=3} \\
= & \frac{\mathrm{Vol}}{(4 \pi t)^{3 / 2}}\left(2-\frac{5 R t}{3}+\frac{20}{3 \sqrt{6 \pi}} R^{3 / 2} t^{3 / 2}-\frac{13 R^{2} t^{2}}{12}\right. \\
& +\frac{8}{3 \sqrt{6 \pi}} R^{5 / 2} t^{5 / 2}-\frac{7}{24} R^{3} t^{3} \\
& +\frac{5 R^{7 / 2} t^{7 / 2}}{9 \sqrt{6 \pi}}-\frac{29}{576} R^{4} t^{4}+\frac{13 R^{9 / 2} t^{9 / 2}}{162 \sqrt{6 \pi}} \\
& \left.-\frac{37 R^{5} t^{5}}{5760}+\mathcal{O}(R t)^{6}\right), \\
\left.\operatorname{Tr}_{2} U_{0}(t, \sigma)\right|_{d=5} & \frac{\operatorname{Vol}^{5 / 2}}{(4 \pi t)^{5 / 2}}-\frac{81 R^{2} t^{2}}{200}+\frac{21 R^{5 / 2} t^{5 / 2}}{25 \sqrt{5 \pi}}-\frac{81 R^{3} t^{3}}{1000} \\
& +\frac{3 R^{7 / 2} t^{7 / 2}}{25 \sqrt{5 \pi}}-\frac{729 R^{4} t^{4}}{80000}+\frac{21 R^{9 / 2} t^{9 / 2}}{2000 \sqrt{5 \pi}} \\
& \left.-\frac{729 R^{5} t^{5}}{1000000}+\mathcal{O}(R t)^{6}\right),
\end{aligned}
$$

which is, again, in agreement with Table 3 . This completes the derivation and checks of heat kernel coefficients on spheres in various integer dimensions.

\subsection{Heat kernels from spectral integrals}

We close with a few observations regarding the usage of the Euler-Maclaurin formula for spectral sums, and spectral integrals for heat kernel coefficients in general dimensions. In integer dimensions, we find that all terms proportional to half-integer powers in $t$, if they arise, originate from the integral term $\int_{a}^{b} \mathrm{~d} \ell f(\ell)$ in the Euler-Maclaurin formula. This observation implies that the remainder $R_{2 n+1}$ do not generate terms proportional to half-integer powers in $t$. Moreover, in odd dimensions it also implies that all heat kernel coefficients $b_{2 n}$ are determined from the expansion of the integral term only, offering the new representation

$b_{2 n}^{(s)}=\left.\frac{(4 \pi)^{d / 2}}{\operatorname{Vol}} \frac{1}{n !} \frac{\mathrm{d}^{n}}{\mathrm{~d} t^{n}}\left(t^{d / 2} \int_{s}^{\infty} \mathrm{d} \ell D_{\ell}^{s} e^{-t \lambda_{\ell}^{s}}\right)\right|_{t=0}$

instead of (84). Note that the spectral sum has become a spectral integral. We rush to add that the expressions (94) for $b_{2 n}$ do not apply in even dimensions, and that there is no analoguous formula for the coefficients $c_{d+2 n}$, the simple reason being that these coefficients receive contributions from both the integral term and from the boundary terms in the Euler-Maclaurin expansion.
In odd dimensions, it is then straightforward to find expressions for heat kernels by evaluating the spectral integral in closed form, followed by a projection onto those terms which in a small $t$ expansion generate half-integer powers in $t$. Following this strategy for scalar heat kernels (which have no contributions from $c_{d+2 n}$ terms), exemplarily in $d=3,5$ and 7 dimensions, we find

$$
\begin{aligned}
& \left.\sum_{n=0}^{\infty} b_{2 n}^{(0)}(E) t^{n}\right|_{d=3}=e^{(E+R / 6) t} \\
& \left.\sum_{n=0}^{\infty} b_{2 n}^{(0)}(E) t^{n}\right|_{d=5}=e^{(E+R / 5) t}\left(1-\frac{R t}{30}\right) \\
& \left.\sum_{n=0}^{\infty} b_{2 n}^{(0)}(E) t^{n}\right|_{d=7}=e^{\left(E+\frac{3}{14} R\right) t}\left(1-\frac{R t}{21}+\frac{4 R^{2} t^{2}}{6615}\right)
\end{aligned}
$$

for arbitrary endomorphism $E$, and in agreement with (31); see also Table 1 . When multiplied with $\frac{\text { Vol }}{(4 \pi t)^{d / 2}},(10)$, it provides closed expressions for the local part of the scalar heat kernels (11). Similar closed expressions for the local heat kernels can be derived in any odd dimension using the spectral integral as discussed above. Note that on compact spaces such as $S^{d}$, the heat kernels also receives non-local "topological" contributions [12]. These are not accounted for in the above.

\subsection{Analytic continuation}

In non-integer dimension, multiplicities are no longer simple polynomials, and closed expressions for the $\ell$ integrals are not available. Still, the coefficients $b_{2 n}$ relate to non-integer powers in $t$ (in non-integer dimensions), and, once more, can only arise from the integral term $\int_{a}^{b} \mathrm{~d} \ell f(\ell)$. This suggests that (94) can be extended to even dimensions by analytic continuation.

We will now explain how spectral integrals can be used in general dimensions to find the heat kernel coefficients $b_{2 n}$. The idea is to evaluate the integral initially in non-integer dimensions using a large $\ell$ expansion for the multiplicities in Table 4 , and interchanging sum and integration. The resulting finite expressions can then be dimensionally continued to integer (even) dimension. ${ }^{1}$ Our procedure also eliminates all contributions to the coefficients $c_{d+2 n}$. In other words, we have

$$
\frac{\mathrm{Vol}}{(4 \pi t)^{d / 2}} \sum_{n} b_{2 n}^{(s)} t^{n}=\left.\int_{0}^{\infty} \mathrm{d} \ell D_{\ell}^{s} e^{-t \lambda_{\ell}^{s}}\right|_{\text {reg. }}
$$

\footnotetext{
${ }^{1}$ Since in all but even dimensions the coefficients $b_{2 n}$ only arise from the spectral integral, it is not surprising that the coefficients $b_{2 n}$ in even dimensions follow from dimensional continuation.
} 
where "reg." indicates the procedure described above. The integration starts at zero for any spin, the reason being that any finite integral $\int_{0}^{s} \mathrm{~d} \ell f(\ell)$ only generates terms in the form of integer powers of $t$ which contribute to the coefficients $c_{d+2 n}$. We apply the procedure in App. C for scalars in general dimensions including all details of the computation. The final result takes the form

$$
\begin{aligned}
& \frac{\mathrm{Vol}}{(4 \pi t)^{d / 2}} \sum_{n} b_{2 n}^{(0)} t^{n}=\frac{1}{\Gamma(d)}\left(\frac{d(d-1)}{R t}\right)^{d / 2} \\
& \quad \times\left\{\sum _ { n = 0 } ^ { \infty } ( \begin{array} { c } 
{ d - 2 } \\
{ 2 n }
\end{array} ) B _ { 2 n } ^ { ( d - 1 ) } ( d - 1 ) \left[I_{d, 2 n}(R t)\right.\right. \\
& \left.\quad-\frac{1}{2}(d-1)^{2} J_{d, 2 n+1}(R t)\right] \\
& +\frac{d-1}{2} \sum_{n=0}^{\infty}\left(\begin{array}{c}
d-2 \\
2 n+1
\end{array}\right) B_{2 n+1}^{(d-1)}(d-1)\left[I_{d, 2 n}(R t)\right. \\
& \left.\left.-2 J_{d, 2 n+1}(R t)\right]\right\}
\end{aligned}
$$

where $B_{n}^{(\ell)}(x)$ are generalised Bernoulli polynomials (C3), and the functions $I_{d, n}(x)$ and $J_{d, n}(x)$ relate to the Kummer hypergeometric series, $(\mathrm{C} 7)$. This result constitutes a representation of heat kernel coefficients which is complementary (yet, equivalent) to the result from the Green's function method (31). A series expansion of (99) in $R$ yields all heat kernel coefficients $b_{2 n}$ in general dimension, and agrees with all findings in integer dimensions reported earlier. In the case where $d$ is an odd integer, the sums can be performed in closed form, and we find agreement with the expressions (95), (96), and (97) in $d=3,5,7$ dimensions.

Finally, we emphasize once more that the spectral integrals with or without dimensional continuation are in general different, $\int_{0}^{\infty} \mathrm{d} \ell D_{\ell}^{s} e^{-t \lambda_{\ell}^{s}} \neq\left.\int_{0}^{\infty} \mathrm{d} \ell D_{\ell}^{s} e^{-t \lambda_{\ell}^{s}}\right|_{\text {reg. }}$. The reason for this is that, without dimensional continuation, the integration additionally generates contributions to the $c_{d+2 n}$ coefficients, all of which are absent in (98). Further, in even dimensions the $b_{2 n}$ coefficients also receive contributions from boundary terms and derivative terms in (85). In contrast to this, the analytical continuation from non-integer dimensions, giving rise to the right-hand side of (98), contains all and only the coefficients $b_{2 n}$ in any dimension.

\section{Discussion}

With the help of Green's functions we have derived general expressions for all heat kernel coefficients of scalars (30), transverse vectors (52), and transverse traceless tensors (69) on the sphere in any dimension and for any endomorphism, also providing the corresponding results for unconstrained fields, see (73), (79). The final expressions are easy to evaluate and straightforward to implement on a practical level, with explicit results stated for selected integer dimensions (Tables 1, 2, 3). Several consistency checks have been performed. We compared the first five heat kernel coefficients and their full dimensional dependence to the known results for heat kernel coefficients on general manifolds [13-21], and found complete agreement.

Furthermore, we have derived the local heat kernel coefficients on spheres from known eigenspectra of Laplacians. The boundedness of remainder terms (88) in the EulerMaclaurin formula has been demonstrated, which is a prerequisite for its applicability to spectral sums. In integer dimensions, full agreement with findings from Green's function is established to high order in the expansion, (89)-(93). We have also found new spectral integral representations (94), (98) for some of the local heat kernel coefficients, and explicit results for scalar heat kernels (99), applicable in general dimension. A virtue of the coefficients from Green's functions (30), (52), and (69) is that they can straightforwardly be extended to non-integer dimension, including for the coefficients $c_{d+2 n}$, a feat which is much harder to achieve using spectral sums.

On a different tack, Green's functions of scalars and transverse vectors on maximally symmetric spaces are of interest for applications in cosmology on de Sitter backgrounds, e.g. $[46,75,75,78]$. As a new addition, we now have derived the Green's function for the Laplacian acting on transverse tensor fields on a fully symmetric background, (59)-(62). We expect that this will be of use in cosmological settings which are sensitive to the graviton propagator.

Finally, our results are of practical relevance for a number of farther reaching applications including the AdS/CFT correspondence for conformal higher spin models [22,23], effective actions for $N=1$ supergravity [24], or trace anomalies [25]. In quantum gravity, possible applications of our results include the perturbative renormalisation of Hořava gravity [26], and tests of the asymptotic safety conjecture where intriguing hints for the near-Gaussianity of gravitational scaling exponents have been observed in $[40,41,43,68]$. In the latter, studies thus far have adopted optimised renormalisation group flows $[32,44]$, which only depend on a few leading heat kernel coefficients [35]. Our findings enable new investigations which are sensitive to many more coefficients without resorting to flat backgrounds and spectral sums or approximations thereof.

Acknowledgements We thank Basem El-Menoufi and Christoph Rahmede for discussions. This work is supported by the Science and Technology Facilities Council (STFC, ST/S505766/1).

Data Availability Statement This manuscript has no associated data or the data will not be deposited. [Authors' comment: No associated data was generated or analysed in this work.] 
Open Access This article is licensed under a Creative Commons Attribution 4.0 International License, which permits use, sharing, adaptation, distribution and reproduction in any medium or format, as long as you give appropriate credit to the original author(s) and the source, provide a link to the Creative Commons licence, and indicate if changes were made. The images or other third party material in this article are included in the article's Creative Commons licence, unless indicated otherwise in a credit line to the material. If material is not included in the article's Creative Commons licence and your intended use is not permitted by statutory regulation or exceeds the permitted use, you will need to obtain permission directly from the copyright holder. To view a copy of this licence, visit http://creativecomm ons.org/licenses/by/4.0/.

Funded by SCOAP ${ }^{3}$.

\section{Appendix A: Expansion coefficients}

In the main body, we encounter expansions of vector and tensor structure functions of the form

$$
S(\sigma)=\sum_{n=0}^{\infty} S_{n} \sigma^{n}
$$

The expansion coefficients $S_{n}$ for the transverse vector Green's function (45) where $S \equiv S^{T}$ and transverse traceless tensor Green's function (63) where $S \equiv S^{T T}$ are required for the computation of heat kernels, see (47) and (64), respectively.

The expansion coefficients of the structure function for transverse vectors are given by where $\gamma$ denotes the Euler-Mascheroni constant and $\psi^{(0)}(x) \equiv$ $\Gamma^{\prime}(x) / \Gamma(x)$ the digamma function. We also recall that the parameter $\bar{\xi}$ is given by

$\bar{\xi}=\frac{1}{2} \sqrt{d\left(-\frac{4(d-1) Q}{R}+d-2\right)+5 .}$

The above expressions are required to obtain the result (48) stated the main text. Note that the dependence of the expansion coefficients (A2) on the Euler-Mascheroni constant and the digamma function drops out in the final expression (48).

For the expansion of the Green's function for transverse traceless tensors, we need to calculate the trace of $G_{Q, \mu \nu \rho^{\prime} \sigma^{\prime}}^{T T}$ in the coincidence limit, see (54). Using the solution (60) for the Green's function of transverse traceless tensors and the corresponding expansion (A1) for the structure function, we develop the term $\mathcal{R}_{\mu \rho^{\prime}} \mathcal{R}_{\nu \sigma^{\prime}} S^{T T}(\sigma)$ up to order eight in $\sigma_{\mu}$. We find

$$
\begin{aligned}
S_{1}^{T}= & \frac{\left(\frac{R}{d(d-1)}\right)^{d / 2-1}}{(4 \pi)^{d / 2} \Gamma\left(\frac{d}{2}\right)(R-d Q)^{2}}\left[\frac{((d-1) d Q-R)}{(d-1) \sin \left(\frac{\pi d}{2}\right)} \cos (\pi \bar{\xi}) \Gamma\left(\frac{d-1}{2}+\bar{\xi}\right) \Gamma\left(\frac{d-1}{2}-\bar{\xi}\right)\right. \\
& \left.-\frac{\Gamma(d-1)(d Q-R)\left(\pi \cot \left(\frac{\pi d}{2}\right)+\psi^{(0)}(d-1)+\gamma-1\right)}{d-3}+\frac{R \Gamma(d-1)}{d-1}\right], \\
S_{2}^{T}= & \frac{2^{-d-1} \pi^{-d / 2}\left(\frac{R}{d(d-1)}\right)^{d / 2-1}}{3(d-1)^{2} d^{3}(d+2) \Gamma\left(\frac{d}{2}\right)\left(Q-\frac{R}{d}\right)^{2}} \\
& \times\left[(5 d-8) R^{2} \Gamma(d-1)+\frac{((d-1) d Q-R)(3(d-1) d Q+2 d R-5 R)}{\sin \left(\frac{\pi d}{2}\right)}\right. \\
& \times \cos (\pi \bar{\xi}) \Gamma\left(\frac{d-1}{2}-\bar{\xi}\right) \Gamma\left(\frac{d-1}{2}+\bar{\xi}\right) \\
& \left.-\frac{R \Gamma(d)(d Q-R)\left(5 d \gamma-8 d+\pi(5 d-8) \cot \left(\frac{\pi d}{2}\right)+(5 d-8) \psi(0)(d-1)-8 \gamma+17\right)}{d-3}\right],
\end{aligned}
$$




$$
\begin{aligned}
\mathcal{R}_{\mu \rho^{\prime}} & \mathcal{R}_{\nu \sigma^{\prime}} S^{T T}(\sigma)=S_{0}^{T T}\left[\left(\frac{R}{2(d-1) d}-\frac{R^{2} \sigma}{12(d-1)^{2} d^{2}}+\frac{R^{3} \sigma^{2}}{180(d-1)^{3} d^{3}}-\frac{R^{4} \sigma^{3}}{5040(d-1)^{4} d^{4}}\right) \sigma_{\mu} \sigma_{\rho^{\prime}} g_{\nu \sigma^{\prime}}\right. \\
& +\left(\frac{R}{2(d-1) d}-\frac{R^{2} \sigma}{12(d-1)^{2} d^{2}}+\frac{R^{3} \sigma^{2}}{180(d-1)^{3} d^{3}}-\frac{R^{4} \sigma^{3}}{5040(d-1)^{4} d^{4}}\right) g_{\mu \rho^{\prime}} \sigma_{\nu} \sigma_{\sigma^{\prime}} \\
& \left.+\left(\frac{R^{2}}{4(d-1)^{2} d^{2}}-\frac{R^{3} \sigma}{12(d-1)^{3} d^{3}}+\frac{R^{4} \sigma^{2}}{80(d-1)^{4} d^{4}}\right) \sigma_{\mu} \sigma_{\rho^{\prime}} \sigma_{\nu} \sigma_{\sigma^{\prime}}+g_{\mu \rho^{\prime}} g_{\nu \sigma^{\prime}}\right] \\
& +S_{1}^{T T}\left[\left(\frac{R \sigma}{2(d-1) d}-\frac{R^{2} \sigma^{2}}{12(d-1)^{2} d^{2}}+\frac{R^{3} \sigma^{3}}{180(d-1)^{3} d^{3}}\right) \sigma_{\mu} \sigma_{\rho^{\prime}} g_{\nu \sigma^{\prime}}\right. \\
& +\left(\frac{R \sigma}{2(d-1) d}-\frac{R^{2} \sigma^{2}}{12(d-1)^{2} d^{2}}+\frac{R^{3} \sigma^{3}}{180(d-1)^{3} d^{3}}\right) g_{\mu \rho^{\prime}} \sigma_{\nu} \sigma_{\sigma^{\prime}} \\
& \left.+\left(\frac{R^{2} \sigma}{4(d-1)^{2} d^{2}}-\frac{R^{3} \sigma^{2}}{12(d-1)^{3} d^{3}}\right) \sigma_{\mu} \sigma_{\rho^{\prime}} \sigma_{\nu} \sigma_{\sigma^{\prime}}+\sigma g_{\mu \rho^{\prime}} g_{\nu \sigma^{\prime}}\right]+S_{2}^{T T}\left[\left(\frac{R \sigma^{2}}{2(d-1) d}-\frac{R^{2} \sigma^{3}}{12(d-1)^{2} d^{2}}\right) \sigma_{\mu} \sigma_{\rho^{\prime}} g_{v \sigma^{\prime}}\right. \\
& \left.+\left(\frac{R \sigma^{2}}{2(d-1) d}-\frac{R^{2} \sigma^{3}}{12(d-1)^{2} d^{2}}\right) g_{\mu \rho^{\prime}} \sigma_{\nu} \sigma_{\sigma^{\prime}}+\frac{R^{2} \sigma^{2}}{4(d-1)^{2} d^{2}} \sigma_{\mu} \sigma_{\rho^{\prime}} \sigma_{\nu} \sigma_{\sigma^{\prime}}+\sigma^{2} g_{\mu \rho^{\prime}} g_{\nu \sigma^{\prime}}\right] \\
& +S_{3}^{T T}\left[\frac{R \sigma^{3}}{2(d-1) d} \sigma_{\mu} \sigma_{\rho^{\prime}} g_{\nu \sigma^{\prime}}+\frac{R \sigma^{3}}{2(d-1) d} g_{\mu \rho^{\prime}} \sigma_{\nu} \sigma_{\sigma^{\prime}}+\sigma^{3} g_{\mu \rho^{\prime}} g_{\nu \sigma^{\prime}}\right]+S_{4}^{T T} \sigma^{4} g_{\mu \rho^{\prime}} g_{\nu \sigma^{\prime}}+\mathcal{O}\left(\sigma_{\alpha}\right)^{9}
\end{aligned}
$$

Expressions for the expansion coefficients $S_{n}^{T T}$ are very long and not given here. Also, acting with the projectors on this expanded term gives long expressions, which are not shown. Similarly to the coefficients (A2), we observe that the coefficients $S_{n}^{T T}$ depend individually on the Euler-Mascheroni constant and the digamma function, whereas the final result (65) is independent thereof. The above expressions are used to arrive at the results (64) and (65) in the main text.

\section{Appendix B: Heat kernels in even dimensions}

In this appendix we supply the first five heat kernel coefficients for scalars, vectors and tensors in even dimensions, where we may combine $b_{2 n}^{(s)}$ and $c_{2 n}^{(s)}$ into a single coefficient by writing

$\hat{b}_{2 n}^{(i)}=b_{2 n}^{(i)}+c_{2 n}^{(i)}$

(recall that $c_{2 n}^{(i)}=0$ for any $n<\frac{d}{2}$ ). Also, since the scalar heat kernel coefficients $c_{2 n}^{(0)}$ vanish identically, we have $\hat{b}_{2 n}^{(0)}=$ $b_{2 n}^{(0)}$, giving

$$
\begin{aligned}
& \hat{b}_{0}^{(0)}=1 \\
& \hat{b}_{3}^{(0)}=\frac{1}{6} R \\
& \hat{b}_{4}^{(0)}=\frac{\left(5 d^{2}-7 d+6\right) R^{2}}{360(d-1) d} \\
& \hat{b}_{6}^{(0)}=\frac{\left(35 d^{4}-112 d^{3}+187 d^{2}-110 d+96\right) R^{3}}{45360(d-1)^{2} d^{2}} \\
& \hat{b}_{8}^{(0)}=\frac{\left(175 d^{6}-945 d^{5}+2389 d^{4}-3111 d^{3}+3304 d^{2}-516 d+2160\right) R^{4}}{5443200(d-1)^{3} d^{3}} .
\end{aligned}
$$

For the transverse vector heat kernels we find

$$
\begin{aligned}
\hat{b}_{0}^{(1)}= & d-1, \\
\hat{b}_{2}^{(1)}= & \frac{\delta_{2, d}}{2} R+\frac{d^{2}-d-6}{6 d} R, \\
\hat{b}_{4}^{(1)}= & \left(\frac{\delta_{2, d}}{4}+\frac{\delta_{4, d}}{24}\right) R^{2} \\
& +\frac{5 d^{4}-12 d^{3}-47 d^{2}-186 d+180}{360(d-1) d^{2}} R^{2}, \\
\hat{b}_{6}^{(1)}= & \left(\frac{\delta_{2, d}}{16}+\frac{\delta_{4, d}}{96}+\frac{\delta_{6, d}}{450}\right) R^{3} \\
& +\frac{35 d^{6}-147 d^{5}-331 d^{4}-3825 d^{3}-676 d^{2}+10992 d-7560}{45360(d-1)^{2} d^{3}} R^{3}, \\
\hat{b}_{8}^{(1)}= & \left(\frac{\delta_{2, d}}{96}+\frac{\delta_{4, d}}{768}+\frac{\delta_{6, d}}{2700}+\frac{15 \delta_{8, d}}{175616}\right) R^{4} \\
& +\frac{R^{4}}{5443200(d-1)^{3} d^{4}}\left(175 d^{8}-1120 d^{7}-866 d^{6}\right. \\
& -38260 d^{5}-31985 d^{4}+34700 d^{3}+405996 d^{2} \\
& -627840 d+226800) .
\end{aligned}
$$


The transverse traceless tensor heat kernels give

$$
\begin{aligned}
\hat{b}_{0}^{(2)}= & \frac{(d-2)(d+1)}{2}, \hat{b}_{2}^{(2)}=3 \delta_{2, d} R+\frac{d^{3}-2 d^{2}-13 d-10}{12(d-1)} R, \\
\hat{b}_{4}^{(2)}= & \left(3 \delta_{2, d}+\frac{5}{8} \delta_{4, d}\right) R^{2}+\frac{5 d^{5}-17 d^{4}-105 d^{3}-475 d^{2}-620 d-228}{720(d-1)^{2} d} R^{2}, \\
\hat{b}_{6}^{(2)}= & \left(\frac{3 \delta_{2, d}}{2}+\frac{5 \delta_{4, d}}{36}+\frac{14 \delta_{6, d}}{225}\right) R^{3}+\frac{35 d^{7}-182 d^{6}-884 d^{5}-8618 d^{4}-21515 d^{3}-23648 d^{2}-38116 d-28032}{90720(d-1)^{3} d^{2}} R^{3}, \\
\hat{b}_{8}^{(2)}= & \left(\frac{\delta_{2, d}}{2}+\frac{5 \delta_{4, d}}{288}+\frac{7 \delta_{6, d}}{1125}+\frac{675 \delta_{8, d}}{175616} R^{4}\right)+\frac{R^{4}}{10886400(d-1)^{4} d^{3}}\left(175 d^{9}-1295 d^{8}-4296 d^{7}\right. \\
& \left.-80514 d^{6}-263073 d^{5}-709635 d^{4}-907534 d^{3}-940876 d^{2}-2454072 d-1896480\right) .
\end{aligned}
$$

Results can now be compared with $[42,48]$ where expressions have been given for the heat kernel coefficients $\hat{b}_{2 n}$ in even dimensions. In $d=4$, our findings for $\hat{b}_{2 i}^{(n)}$ agree numerically with the corresponding expressions $\left.b_{2 i}\right|_{n}$ given in [48] and in appendix $B$ of [42], except for $\left.b_{8}\right|_{1}$. For general $d$, deviations appear in the algebraic expressions for $\left.b_{8}\right|_{1}$ and $\left.b_{8}\right|_{2}$, and some contributions which uniquely arise in even integer dimensions (proportional to Kronecker deltas) have been missed. Our results are consistent with the heat kernel, spectral sums, and expressions for general backgrounds as found in the literature.

\section{Appendix C: Spectral integrals}

In this appendix we detail the derivation of spectral integrals and heat kernel coefficients from spectral sums in general dimension, using dimensional continuation as outlined in Sect. 4.4. We focus on scalars for simplicity. The computational steps for transverse vectors and transverse traceless tensors are the same and only differ by the specific form of the multiplicities.

We start by taking the dimension $d$ to be non-integer. Results for integer dimension then follow from dimensional continuation. Basic input are the eigenvalues and their multiplicities (see Table 4), which for scalars are given by

$D_{\ell}^{0}=\frac{(2 \ell+d-1) \Gamma(\ell+d-1)}{\Gamma(d) \Gamma(\ell+1)}$.

A large $\ell$ expansion is performed using the asymptotic series [84]

$\frac{\Gamma(\ell+a)}{\Gamma(\ell+b)} \simeq \ell^{a-b} \sum_{n=0}^{\infty} \frac{1}{\ell^{n}}\left(\begin{array}{c}a-b \\ n\end{array}\right) B_{n}^{(a-b+1)}(a)$,

where the " $\simeq$ " indicates the asymptotic nature of the series. The generalised Bernoulli polynomials $B_{n}^{(\ell)}(x)$ are defined as the Taylor coefficients of

$$
\left(\frac{t}{e^{t}-1}\right)^{\ell} e^{x t}=\sum_{n=0}^{\infty} B_{n}^{(\ell)}(x) \frac{t^{n}}{n !} .
$$

For the particular case $(\mathrm{C} 1)$, this gives us

$$
D_{\ell}^{0} \simeq \frac{2 \ell+d-1}{\Gamma(d)} \sum_{n=0}^{\infty} \ell^{d-2-n}\left(\begin{array}{c}
d-2 \\
n
\end{array}\right) B_{n}^{(d-1)}(d-1) .
$$

We now have to perform the spectral integral

$$
\begin{gathered}
\int_{0}^{\infty} \mathrm{d} \ell D_{\ell}^{0} e^{-\lambda_{\ell}^{0} t} \simeq \frac{1}{\Gamma(d)} \sum_{n=0}^{\infty}\left(\begin{array}{c}
d-2 \\
n
\end{array}\right) B_{n}^{(d-1)}(d-1) \\
\int_{0}^{\infty} \mathrm{d} \ell \ell^{d-2-n}(2 \ell+d-1) e^{-\frac{\ell(\ell+d-1)}{d(d-1)} R t},
\end{gathered}
$$

which can be done term by term with the help of

$$
\begin{aligned}
& \int_{0}^{\infty} \mathrm{d} \ell \ell^{d-1-n} e^{-\frac{\ell(\ell+d-1)}{d(d-1)} R t} \\
& \quad=\frac{1}{2}\left(\frac{R t}{d(d-1)}\right)^{-d / 2}\left[I_{d, n}(R t)-(d-1) J_{d, n}(R t)\right],
\end{aligned}
$$

where we have introduced the shorthand notations

$$
\begin{aligned}
I_{d, n}(x)= & \left(\frac{x}{d(d-1)}\right)^{n / 2} \Gamma\left(\frac{d-n}{2}\right) \\
& \times{ }_{1} F_{1}\left(\frac{d-n}{2}, \frac{1}{2}, \frac{d-1}{4 d} x\right) \\
J_{d, n}(x)= & \left(\frac{x}{d(d-1)}\right)^{(n+1) / 2} \Gamma\left(\frac{d-n+1}{2}\right) \\
& \times{ }_{1} F_{1}\left(\frac{d-n+1}{2}, \frac{3}{2}, \frac{d-1}{4 d} x\right)
\end{aligned}
$$

involving the Kummer function ${ }_{1} F_{1}(a, b, z)=\sum_{k=0}^{\infty} \frac{a_{(k)}}{b_{(k}} \frac{z^{k}}{k !}$, and $a_{(k)}$ the rising factorial. The expression (C6) with (C7) 
is valid for general $d>1, R t>0$, and $n<d$. By analytic continuation in the dimension we extend its domain of validity to all $n$. The right-hand side of (C6) contains poles from Gamma functions provided $d-n$ is a negative integer or zero. However, these poles will be multiplied by zeros from the binomial coefficient in (C4), giving a finite result for any $d-n$ if analytically continued from non-integer $d$. Following this strategy, we find

$$
\begin{aligned}
\left.\int_{0}^{\infty} \mathrm{d} \ell D_{\ell}^{0} e^{-\lambda_{\ell}^{0} t}\right|_{\text {reg. }} \\
=\frac{2}{\Gamma(d)}\left(\frac{d(d-1)}{R t}\right)^{d / 2} \sum_{n=0}^{\infty}\left(\begin{array}{c}
d-2 \\
n
\end{array}\right) B_{n}^{(d-1)}(d-1) \\
\quad \times\left[\frac{1}{2} I_{d, n}(R t)-\frac{d-1}{2} J_{d, n}(R t)\right. \\
\left.\quad+\frac{d-1}{4} I_{d, n+1}(R t)-\frac{(d-1)^{2}}{4} J_{d, n+1}(R t)\right]
\end{aligned}
$$

where "reg." indicates our procedure of analytic continuation and the usage of an asymptotic large $\ell$ expansion. Note that the right-hand side contains terms proportional to $t^{-d / 2+m}$ and $t^{-(d+2 m+1) / 2}$ for any integer $m \geq 0$. The former relate to the heat kernel coefficients $b_{2 m}$. The latter cannot arise from a heat kernel expansion, and, hence, their coefficients must vanish identically. With this in mind, we write (C8) as

$$
\begin{aligned}
& \left.\int_{0}^{\infty} \mathrm{d} \ell D_{\ell}^{0} e^{-\lambda_{\ell}^{0} t}\right|_{\text {reg. }}=\frac{1}{\Gamma(d)}\left(\frac{d(d-1)}{R t}\right)^{d / 2} \\
& \quad \times\left\{\sum _ { n = 0 } ^ { \infty } ( \begin{array} { c } 
{ d - 2 } \\
{ 2 n }
\end{array} ) B _ { 2 n } ^ { ( d - 1 ) } ( d - 1 ) \left[I_{d, 2 n}(R t)\right.\right. \\
& \left.\quad-\frac{1}{2}(d-1)^{2} J_{d, 2 n+1}(R t)\right] \\
& +\frac{d-1}{2} \sum_{n=0}^{\infty}\left(\begin{array}{c}
d-2 \\
2 n+1
\end{array}\right) B_{2 n+1}^{(d-1)}(d-1)\left[I_{d, 2 n}(R t)\right. \\
& \left.\left.\quad-2 J_{d, 2 n+1}(R t)\right]\right\}
\end{aligned}
$$

which manifestly contains only terms of the form $\sim t^{-d / 2+m}$. Together with (98) this gives rise to the result (99) in the main text.

As a final check, we confirm that any terms of the form $t^{-(d+2 m+1) / 2}$ in (C8) vanish, as they must. Their sum can be written as

$$
\begin{aligned}
0= & \frac{d-1}{2} \sum_{n=0}^{\infty}\left(\begin{array}{c}
d-2 \\
2 n
\end{array}\right) B_{2 n}^{(d-1)}(d-1)\left[I_{d, 2 n+1}(R t)\right. \\
& \left.-2 J_{d, 2 n}(R t)\right]
\end{aligned}
$$

$$
\begin{aligned}
& +\sum_{n=0}^{\infty}\left(\begin{array}{c}
d-2 \\
2 n+1
\end{array}\right) B_{2 n+1}^{(d-1)}(d-1)\left[I_{d, 2 n+1}(R t)\right. \\
& \left.-\frac{1}{2}(d-1)^{2} J_{d, 2 n}(R t)\right]
\end{aligned}
$$

and we have checked the validity of (C10) for general dimension through a series expansion in curvature $R$ up to $\mathcal{O}\left(R^{10}\right)$. We expect this to hold true to any order.

The same steps which have led to (C9) can be repeated for transverse vectors and transverse traceless tensors leading to corresponding expressions.

\section{References}

1. V. Fock, Proper time in classical and quantum mechanics. Phys. Z. Sowjetunion 12, 404 (1937)

2. J.S. Schwinger, On gauge invariance and vacuum polarization. Phys. Rev. 82, 664 (1951). https://doi.org/10.1103/PhysRev.82. 664

3. B.S. DeWitt, Quantum theory of gravity. 1. The canonical theory. Phys. Rev. 160, 1113 (1967). https://doi.org/10.1103/PhysRev. 160.1113

4. B.S. DeWitt, Quantum theory of gravity. 2. The manifestly covariant theory. Phys. Rev. 162, 1195 (1967). https://doi.org/10.1103/ PhysRev.162.1195

5. B.S. DeWitt, Quantum theory of gravity. 3. Applications of the covariant theory. Phys. Rev. 162, 1239 (1967). https://doi.org/10. 1103/PhysRev.162.1239

6. D. Vassilevich, Heat kernel expansion: user's manual. Phys. Rep. 388, 279 (2003). https://doi.org/10.1016/j.physrep.2003.09.002. arXiv:hep-th/0306138

7. M. Bordag, U. Mohideen, V.M. Mostepanenko, New developments in the Casimir effect. Phys. Rep. 353, 1 (2001). https://doi.org/10. 1016/S0370-1573(01)00015-1. arXiv:quant-ph/0106045

8. A.O. Barvinsky, G.A. Vilkovisky, The generalized SchwingerDewitt technique in gauge theories and quantum gravity. Phys. Rep. 119, 1 (1985). https://doi.org/10.1016/0370-1573(85)90148-6

9. I.G. Avramidi, Heat kernel and quantum gravity. Lect. Notes Phys. Monogr. 64, 1 (2000). https://doi.org/10.1007/3-540-46523-5

10. N.D. Birrell, P.C.W. Davies, Quantum Fields in Curved Space. Cambridge Monographs on Mathematical Physics (Cambridge University Press, Cambridge, 1984). https://doi.org/10.1017/ CBO9780511622632

11. E. Fradkin, A.A. Tseytlin, One loop effective potential in gauged O(4) supergravity. Nucl. Phys. B 234, 472 (1984). https://doi.org/ 10.1016/0550-3213(84)90074-9

12. R. Camporesi, Harmonic analysis and propagators on homogeneous spaces. Phys. Rep. 196, 1 (1990). https://doi.org/10.1016/ 0370-1573(90)90120-Q

13. B.S. DeWitt, Dynamical theory of groups and fields. Conf. Proc. C630701, 585 (1964)

14. P.B. Gilkey, The spectral geometry of a Riemannian manifold. J. Differ. Geom. 10, 601 (1975)

15. S.M. Christensen, Vacuum expectation value of the stress tensor in an arbitrary curved background: the covariant point separation method. Phys. Rev. D 14, 2490 (1976). https://doi.org/10.1103/ PhysRevD.14.2490

16. S.M. Christensen, Regularization, renormalization, and covariant geodesic point separation. Phys. Rev. D 17, 946 (1978). https://doi. org/10.1103/PhysRevD.17.946 
17. P. Amsterdamski, A.L. Berkin, D.J. O'Connor, B(8) 'Hamidew' coefficient for a scalar field. Class. Quantum Gravity 6, 1981 (1989). https://doi.org/10.1088/0264-9381/6/12/024

18. I.G. Avramidi, Background field calculations in quantum field theory (vacuum polarization). Theor. Math. Phys. 79, 494 (1989). https://doi.org/10.1007/BF01016530

19. I.G. Avramidi, The covariant technique for calculation of one loop effective action. Nucl. Phys. B 355, 712 (1991). https://doi.org/10.1016/0550-3213(91)90492-G. https://doi.org/ 10.1016/S0550-3213(97)00717-7

20. I.G. Avramidi, The covariant technique for the calculation of the heat kernel asymptotic expansion. Phys. Lett. B 238, 92 (1990). https://doi.org/10.1016/0370-2693(90)92105-R

21. A.E.M. van de Ven, Index free heat kernel coefficients. Class. Quantum Gravity 15, 2311 (1998). https://doi.org/10.1088/0264-9381/ 15/8/014. arXiv:hep-th/9708152

22. S. Giombi, I.R. Klebanov, One loop tests of higher spin AdS/CFT. JHEP 12, 068 (2013). https://doi.org/10.1007/JHEP12(2013)068. arXiv: 1308.2337

23. S. Giombi, I.R. Klebanov, A.A. Tseytlin, Partition functions and casimir energies in higher spin $\mathrm{AdS}_{d+1} / \mathrm{CFT}_{d}$. Phys. Rev. D 90, 024048 (2014). https://doi.org/10.1103/PhysRevD.90.024048. arXiv: 1402.5396

24. J.R. David, M.R. Gaberdiel, R. Gopakumar, The heat kernel on AdS(3) and its applications. JHEP 04, 125 (2010). https://doi.org/ 10.1007/JHEP04(2010)125. arXiv:0911.5085

25. F. Bastianelli, O. Corradini, E. Vassura, Quantum mechanical path integrals in curved spaces and the type-A trace anomaly. JHEP 04, 050 (2017). https://doi.org/10.1007/JHEP04(2017)050. arXiv: 1702.04247

26. A.O. Barvinsky, D. Blas, M. Herrero-Valea, D.V. Nesterov, G. Pérez-Nadal, C.F. Steinwachs, Heat kernel methods for Lifshitz theories. JHEP 06, 063 (2017). https://doi.org/10.1007/ JHEP06(2017)063. arXiv:1703.04747

27. S. Weinberg, Ultraviolet divergences in quantum theories of gravitation, in General Relativity: An Einstein centenary survey, ed. by S.W. Hawking, W. Israel (Cambridge University Press, Cambridge, 1979), p. 790

28. D.F. Litim, Fixed points of quantum gravity and the renormalisation group. arXiv:0810.3675

29. M. Reuter, Nonperturbative evolution equation for quantum gravity. Phys. Rev. D 57, 971 (1998). https://doi.org/10.1103/ PhysRevD.57.971. arXiv:hep-th/9605030

30. W. Souma, Nontrivial ultraviolet fixed point in quantum gravity. Prog. Theor. Phys. 102, 181 (1999). https://doi.org/10.1143/PTP. 102.181. arXiv:hep-th/9907027

31. O. Lauscher, M. Reuter, Flow equation of quantum Einstein gravity in a higher- derivative truncation. Phys. Rev. D 66, 025026 (2002). https://doi.org/10.1103/PhysRevD.66.025026. arXiv:hep-th/0205062

32. D.F. Litim, Fixed points of quantum gravity. Phys. Rev. Lett. 92, 201301 (2004). https://doi.org/10.1103/PhysRevLett.92.201301. arXiv:hep-th/0312114

33. P. Fischer, D.F. Litim, Fixed points of quantum gravity in extra dimensions. Phys. Lett. B 638, 497 (2006). https://doi.org/10.1016/ j.physletb.2006.05.073. arXiv:hep-th/0602203

34. P.F. Machado, F. Saueressig, On the renormalization group flow of f(R)-gravity. Phys. Rev. D 77, 124045 (2008). https://doi.org/10. 1103/PhysRevD.77.124045. arXiv:0712.0445

35. A. Codello, R. Percacci, C. Rahmede, Investigating the ultraviolet properties of gravity with a Wilsonian renormalization group equation. Ann. Phys. 324, 414 (2009). https://doi.org/10.1016/j. aop.2008.08.008. arXiv:0805.2909

36. D. Benedetti, P.F. Machado, F. Saueressig, Asymptotic safety in higher-derivative gravity. Mod. Phys. Lett. A 24, 2233 (2009). https://doi.org/10.1142/S0217732309031521. arXiv:0901.2984
37. M.R. Niedermaier, Gravitational fixed points from perturbation theory. Phys. Rev. Lett. 103, 101303 (2009). https://doi.org/10. 1103/PhysRevLett.103.101303

38. D. Benedetti, F. Caravelli, The Local potential approximation in quantum gravity. JHEP 06, 017 (2012). https://doi.org/10.1007/ JHEP06(2012)017, https://doi.org/10.1007/JHEP10(2012)157. arXiv: 1204.3541

39. N. Christiansen, D.F. Litim, J.M. Pawlowski, A. Rodigast, Fixed points and infrared completion of quantum gravity. Phys. Lett. B 728, 114 (2014). https://doi.org/10.1016/j.physletb.2013.11.025. arXiv: 1209.4038

40. K. Falls, D.F. Litim, K. Nikolakopoulos, C. Rahmede, A bootstrap towards asymptotic safety. arXiv:1301.4191

41. K. Falls, D.F. Litim, K. Nikolakopoulos, C. Rahmede, Further evidence for asymptotic safety of quantum gravity. Phys. Rev. D 93, 104022 (2016). https://doi.org/10.1103/PhysRevD.93.104022. arXiv: 1410.4815

42. K. Falls, C.R. King, D.F. Litim, K. Nikolakopoulos, C. Rahmede, Asymptotic safety of quantum gravity beyond Ricci scalars. Phys. Rev. D 97, 086006 (2018). https://doi.org/10.1103/PhysRevD.97. 086006. arXiv:1801.00162

43. K.G. Falls, D.F. Litim, J. Schröder, Aspects of asymptotic safety for quantum gravity. Phys. Rev. D 99, 126015 (2019). https://doi. org/10.1103/PhysRevD.99.126015. arXiv: 1810.08550

44. D.F. Litim, Optimized renormalization group flows. Phys. Rev. D 64, 105007 (2001). https://doi.org/10.1103/PhysRevD.64.105007. arXiv:hep-th/0103195

45. J.S. Dowker, R. Critchley, Effective Lagrangian and energy momentum tensor in de Sitter space. Phys. Rev. D 13, 3224 (1976). https://doi.org/10.1103/PhysRevD.13.3224

46. N.C. Tsamis, R.P. Woodard, A maximally symmetric vector propagator. J. Math. Phys. 48, 052306 (2007). https://doi.org/10.1063/ 1.2738361. arXiv:gr-qc/0608069

47. G. Narain, Green's function of the Vector fields on DeSitter Background. arXiv:1408.6193

48. C. Rahmede, Investigating the ultraviolet properties of gravity with a wilsonian renormalization group equation (SISSA, Trieste, 2008). Ph.D. thesis

49. R. Percacci, An Introduction to Covariant Quantum Gravity and Asymptotic Safety, vol. 3 of 100 Years of General Relativity (World Scientific, Singapore, 2017). https://doi.org/10.1142/10369

50. S. Christensen, M. Duff, Quantizing gravity with a cosmological constant. Nucl. Phys. B 170, 480 (1980). https://doi.org/10.1016/ 0550-3213(80)90423-X

51. G.W. Gibbons, M.J. Perry, Quantizing gravitational instantons. Nucl. Phys. B 146, 90 (1978). https://doi.org/10.1016/ 0550-3213(78)90434-0

52. G. Gibbons, S. Hawking, M. Perry, Path integrals and the indefiniteness of the gravitational action. Nucl. Phys. B 138, 141 (1978). https://doi.org/10.1016/0550-3213(78)90161-X

53. D.F. Litim, J.M. Pawlowski, On gauge invariant Wilsonian flows, in The exact renormalization group. Proceedings, Workshop, Faro, Portugal, September 10-12, 1998, pp 168-185. arXiv:hep-th/9901063

54. J. Berges, N. Tetradis, C. Wetterich, Non-perturbative renormalization flow in quantum field theory and statistical physics. Phys. Rep. 363, 223 (2002). https://doi.org/10.1016/ S0370-1573(01)00098-9. arXiv:hep-ph/0005122

55. D.F. Litim, Renormalisation group and the Planck scale. Philos. Trans. R. Soc. Lond. A369, 2759 (2011). https://doi.org/10.1098/ rsta.2011.0103. arXiv: 1102.4624

56. D.F. Litim, J.M. Pawlowski, Perturbation theory and renormalization group equations. Phys. Rev. D 65, 081701 (2002). https://doi. org/10.1103/PhysRevD.65.081701. arXiv:hep-th/0111191

57. D.F. Litim, J.M. Pawlowski, Completeness and consistency of renormalisation group flows. Phys. Rev. D 66, 
025030 (2002). https://doi.org/10.1103/PhysRevD.66.025030. arXiv:hep-th/0202188

58. J.M. Pawlowski, Aspects of the functional renormalisation group. Ann. Phys. 322, 2831 (2007). https://doi.org/10.1016/j.aop.2007. 01.007. arXiv:hep-th/0512261

59. M. Reuter, F. Saueressig, Quantum Einstein gravity. New J. Phys. 14, 055022 (2012). https://doi.org/10.1088/1367-2630/14/ 5/055022. arXiv: 1202.2274

60. D.F. Litim, Mind the gap. Int. J. Mod. Phys. A 16, 2081 (2001). https://doi.org/10.1142/S0217751X01004748. arXiv:hep-th/0104221

61. D.F. Litim, D. Zappala, Ising exponents from the functional renormalisation group. Phys. Rev. D 83, 085009 (2011). https://doi.org/ 10.1103/PhysRevD.83.085009. arXiv:1009.1948

62. I. Balog, H. Chaté, B. Delamotte, M. Marohnic, N. Wschebor, Convergence of non-perturbative approximations to the renormalization group. arXiv:1907.01829

63. R. Floreanini, R. Percacci, The Heat kernel and the average effective potential. Phys. Lett. B 356, 205 (1995). https://doi.org/10. 1016/0370-2693(95)00799-Q. arXiv:hep-th/9505172

64. D. Dou, R. Percacci, The running gravitational couplings. Class. Quantum Gravity 15, 3449 (1998). https://doi.org/10.1088/ 0264-9381/15/11/011. arXiv:hep-th/9707239

65. F. Freire, D.F. Litim, J.M. Pawlowski, Gauge invariance and background field formalism in the exact renormalization group. Phys. Lett. B 495, 256 (2000). https://doi.org/10.1016/ S0370-2693(00)01231-4. arXiv:hep-th/0009110

66. D.F. Litim, Optimization of the exact renormalization group. Phys. Lett. B 486, 92 (2000). https://doi.org/10.1016/ S0370-2693(00)00748-6. arXiv:hep-th/0005245

67. D.F. Litim, J.M. Pawlowski, Non-perturbative thermal flows and resummations. JHEP 11, 026 (2006). https://doi.org/10.1088/ 1126-6708/2006/11/026. arXiv:hep-th/0609122

68. K. Falls, D.F. Litim, K. Nikolakopoulos, C. Rahmede, On de Sitter solutions in asymptotically safe $f(R)$ theories. Class. Quantum Gravity 35, 135006 (2018). https://doi.org/10.1088/1361-6382/ aac440. arXiv: 1607.04962

69. D. Benedetti, P.F. Machado, F. Saueressig, Taming perturbative divergences in asymptotically safe gravity. Nucl. Phys. B 824, 168 (2010). https://doi.org/10.1016/j.nuclphysb.2009.08.023. arXiv:0902.4630

70. K. Falls, N. Ohta, Renormalization group equation for $f(R)$ gravity on hyperbolic spaces. Phys. Rev. D 94, 084005 (2016). https://doi. org/10.1103/PhysRevD.94.084005. arXiv:1607.08460

71. D. Benedetti, Critical behavior in spherical and hyperbolic spaces. J. Stat. Mech. 1501, P01002 (2015). https://doi.org/10.1088/ 1742-5468/2015/01/P01002. arXiv:1403.6712
72. A. Codello, R. Percacci, L. Rachwał, A. Tonero, Computing the effective action with the functional renormalization group. Eur. Phys. J. C 76, 226 (2016). https://doi.org/10.1140/epjc/ s10052-016-4063-3. arXiv:1505.03119

73. A. Bonanno, M. Reuter, Proper time flow equation for gravity. JHEP 02, 035 (2005). https://doi.org/10.1088/1126-6708/2005/ 02/035. arXiv:hep-th/0410191

74. B. Allen, T. Jacobson, Vector two point functions in maximally symmetric spaces. Commun. Math. Phys. 103, 669 (1986). https:// doi.org/10.1007/BF01211169

75. S.P. Miao, N.C. Tsamis, R.P. Woodard, The graviton propagator in de donder gauge on de Sitter background. J. Math. Phys. 52, 122301 (2011). https://doi.org/10.1063/1.3664760. arXiv:1106.0925

76. E.W. Weisstein, "Hypergeometric Function." MathWorldA Wolfram Web Resource. http://mathworld.wolfram.com/ HypergeometricFunction.html. Accessed 9 June 2019

77. F.W.J. Olver, A.B. Olde Daalhuis, D.W. Lozier, B.I. Schneider, R.F. Boisvert, C.W. Clark et al., "Eq. 5.11.8." NIST Digital Library of Mathematical Functions. https://dlmf.nist.gov/5.11.E8. Accessed 9 June 2019

78. P.J. Mora, N.C. Tsamis, R.P. Woodard, Graviton propagator in a general invariant gauge on de Sitter. J. Math. Phys. 53, 122502 (2012). https://doi.org/10.1063/1.4764882. arXiv:1205.4468

79. E. Mottola, Functional integration over geometries. J. Math. Phys. 36, 2470 (1995). https://doi.org/10.1063/1.531359. arXiv:hep-th/9502109

80. O. Lauscher, M. Reuter, Ultraviolet fixed point and generalized flow equation of quantum gravity. Phys. Rev. D 65, 025013 (2002). https://doi.org/10.1103/PhysRevD.65.025013. arXiv:hep-th/0108040

81. M.A. Rubin, C.R. Ordonez, Eigenvalues and degeneracies for $n$ dimensional tensor spherical harmonics. J. Math. Phys. 25, 2888 (1984). https://doi.org/10.1063/1.526034

82. M.A. Rubin, C.R. Ordonez, Symmetric tensor eigen spectrum of the Laplacian on $n$ spheres. J. Math. Phys. 26, 65 (1985). https:// doi.org/10.1063/1.526749

83. M. Demmel, F. Saueressig, O. Zanusso, RG flows of quantum Einstein gravity on maximally symmetric spaces. JHEP 06, 026 (2014). https://doi.org/10.1007/JHEP06(2014)026. arXiv:1401.5495

84. F.W.J. Olver, A.B. Olde Daalhuis, D.W. Lozier, B.I. Schneider, R.F. Boisvert, C.W. Clark et al., "Eq. 5.11.e13.” NIST Digital Library of Mathematical Functions. http://dlmf.nist.gov/5.11.E13. Accessed $24 \mathrm{Feb} 2020$ 